\title{
Empirical Temperature Measurement in Protoplanetary Disks
}

\author{
Erik Weaver, Andrea Isella (iD, and Yann Boehler(iD) \\ Department of Physics and Astronomy, Rice University, 6100 Main Street, MS-108, Houston, TX 77005 USA; etw1@ @ice.edu \\ Received 2017 November 9; revised 2017 December 15; accepted 2017 December 26; published 2018 January 30
}

\begin{abstract}
The accurate measurement of temperature in protoplanetary disks is critical to understanding many key features of disk evolution and planet formation, from disk chemistry and dynamics, to planetesimal formation. This paper explores the techniques available to determine temperatures from observations of single, optically thick molecular emission lines. Specific attention is given to issues such as the inclusion of optically thin emission, problems resulting from continuum subtraction, and complications of real observations. Effort is also made to detail the exact nature and morphology of the region emitting a given line. To properly study and quantify these effects, this paper considers a range of disk models, from simple pedagogical models to very detailed models including full radiative transfer. Finally, we show how the use of the wrong methods can lead to potentially severe misinterpretations of data, leading to incorrect measurements of disk temperature profiles. We show that the best way to estimate the temperature of emitting gas is to analyze the line peak emission map without subtracting continuum emission. Continuum subtraction, which is commonly applied to observations of line emission, systematically leads to underestimation of the gas temperature. We further show that once observational effects such as beam dilution and noise are accounted for, the line brightness temperature derived from the peak emission is reliably within $10 \%-15 \%$ of the physical temperature of the emitting region, assuming optically thick emission. The methodology described in this paper will be applied in future works to constrain the temperature, and related physical quantities, in protoplanetary disks observed with ALMA.
\end{abstract}

Key words: circumstellar matter - planets and satellites: formation - protoplanetary disks

\section{Introduction}

Temperature is one of the key parameters describing the conditions and dynamics inside protoplanetary disks. It controls disk chemistry, and by setting the locations of various snow lines (Qi et al. 2013), it affects disk structure. Thermal motion is a major component of gas dynamics (Beckwith \& Sargent 1993) and must be fully understood in order to determine the extent of any nonthermal turbulent motion present (Teague et al. 2016; Flaherty et al. 2017). Temperature also affects the growth of dust grains, and by extension, the growth of planetesimals (Blum \& Wurm 2008; Williams \& Cieza 2011; Testi et al. 2014). Lastly, temperature controls how strongly and at which frequencies different components of the disk emit.

The calculation of temperature in disks is nontrivial. Current methods rely on radiative transfer codes, which use either Monte Carlo methods to simulate billions of photons propagating through the disk to determine a temperature at each point or use approximations to analytically solve the equation of radiative transfer. (See Chiang \& Goldreich 1997; Rafikov \& De Colle 2006; Dullemond et al. 2007.) Energy is emitted primarily by the central star, though contributions from external stellar radiation and, occasionally, viscous heating are included. By modeling the dust and gas emission and comparing to observation, the thermal structure of the disk can be estimated. (See, for example, Piétu et al. 2007; Rosenfeld et al. 2013.) Unfortunately, this process of forward modeling is time consuming, computationally expensive, and relies on assumptions, such as the vertical dust distribution of the disk and dust opacity.

Temperatures can instead be inferred from optically thick molecular emission lines. Spectral lines from molecules have long been powerful tools in radio astronomy, and in the current era of high-resolution ALMA observations, offer unprecedented information. The bulk of disk mass is in molecular hydrogen, which, lacking a dipole moment, does not readily emit. However, other molecules, such as $\mathrm{CO}$ and $\mathrm{CN}$, are abundant in circumstellar disks, emit strong spectral lines, and can be used to trace the distribution of molecular hydrogen (Guilloteau et al. 2013). Molecular lines such as CO rotational transitions are observable from ground-based telescopes, such as ALMA. ALMA's ability to offer observations with subarcsecond angular resolution allows the disk structure to be spatially resolved. Additionally, because these lines can be optically thick, the spatial temperature distribution can be determined.

Observation of a source yields a map of the surface brightness, which can be turned into a map of temperature via the Planck equation. Brightness temperature can be calculated from maps of molecular lines, for example, the rotational transitions of $\mathrm{CO}$. Other examples include $\mathrm{NH}_{3}$, which can be used to measure temperatures of molecular cloud cores, and $\mathrm{HCO}^{+}$. (See van Zadelhoff et al. 2001; Juvela et al. 2012; Yen et al. 2016). While the process of determining a brightness temperature can be quite simple, there are a number of subtleties that can affect the interpretations in terms of physical gas temperature. Under certain conditions, problems can arise due to the subtraction of continuum emission, inclusion of optically thin emission, and complications from beam dilution. This paper will explore the conditions under which these problems arise and what alternate techniques can be employed to deal with them.

First, observations typically have the continuum emission subtracted off in order to determine the pure line flux. The problem is that this process can end up removing line emission as well as the continuum. This comes about because the continuum emission is not a constant value across the 
spectrum, as normally assumed, because the line absorbs the continuum. At the brightest points on the line spectrum, the continuum emission is at its weakest, and for strong lines, can be almost entirely attenuated. This means that if the continuum emission is bright, much of the line emission can be subtracted off, and any temperature measured will be too low, as will any flux measured. The second problem is that no line is entirely optically thick. While the line center may be bright and wholly optically thick, the line wings quickly become optically thin, and when the spectrum is integrated to produce the integrated emission map, this thin emission is included, causing another underestimate. Lastly, the beam size and finite sensitivity of observations mean that the ability to determine the temperature is affected by both beam dilution and noise. Beam dilution occurs when the beam size is larger than the spatial structure of the source and leads to an underestimate, while noise can lead to both under- and overestimates. These issues come about in various different situations and can potentially have severe effects on analyses. The goal of our study is to quantify these effects for typical protoplanetary disks.

The structure of the paper is as follows. Section 2 details a pedagogical model suitable for demonstrating the basic concepts and methods. Section 3 then repeats the analysis for a realistic disk model. Section 4 describes the results of the simulated observation with ALMA, and our conclusions and summary can be found in Section 5 .

\section{Pedagogical Model}

\subsection{Structure and Emission}

In this section, we discuss the derivation of the temperature and density of a circumstellar disk based on a simple disk model with the following properties. We assume a disk of total mass $M_{d}$ distributed following a center-symmetric power-law surface density,

$$
\Sigma(r)=\Sigma_{0} \times\left(\frac{r}{r_{0}}\right)^{-p}
$$

between an inner radius $\left(r_{\min }\right)$ of 0.1 au and an outer radius $\left(r_{\max }\right)$ of $100 \mathrm{au} . \Sigma_{0}$ is the surface density normalization at the reference radius $r_{0}$. In this paper, $r_{0}$ is taken to be $10 \mathrm{au}$, and the power-law index, $p$, is taken to be $1 . \Sigma_{0}$ is determined from the disk mass via

$$
\Sigma_{0}=\frac{(2-p) \cdot M_{d}}{2 \pi r_{0}^{p}\left(r_{\max }^{p}-r_{\min }^{p}\right)} .
$$

The disk temperature distribution is also a radial power law with an index of -0.5 and is vertically isothermal. Furthermore, the disk temperature does not depend on the disk mass. This is only an approximation, but it reasonably models the interior of the disk (see, e.g., Dullemond et al. 2001), and as discussed below, allows us to solve the radiative transfer equation for the dust and gas emission analytically. The temperature profile is

$$
T(r)=70 \mathrm{~K} \times\left(\frac{r}{r_{0}}\right)^{-0.5} .
$$

This is chosen to roughly match the temperature profile resulting from a solar-type star. The gas density $\rho(r, z)$ is calculated assuming that the disk is in hydrostatic equilibrium.
Since the disk is vertically isothermal, the density is

$$
\rho(r, z)=\frac{\Sigma(r)}{\sqrt{2 \pi} h(r)} \exp \left(\frac{-z^{2}}{2 h^{2}(r)}\right),
$$

and the pressure scale height is $h(r)=c_{s}(r) / \Omega(r)$, where $c_{s}(r)=\sqrt{\gamma k_{b} T(r) / \mu m_{p}}$ is the gas sound speed and $\Omega(r)=$ $\sqrt{G M_{\star} / r^{3}}$ is the Keplerian angular velocity. Using a mean molecular weight $\mu=2.3$; an adiabatic index, $\gamma=7 / 5$, appropriate for molecular hydrogen; Boltzmann constant $k_{b}=$ $1.38 \times 10^{-16} \mathrm{erg} \mathrm{K}^{-1}$; a proton mass $m_{p}=1.67 \times 10^{-24} \mathrm{~g}$; a gravitational constant $G=6.67 \times 10^{-8} \mathrm{~cm}^{3} \mathrm{~g}^{-1} \mathrm{~s}^{-2}$; and a stellar mass $M_{\star}=2 \times 10^{33} \mathrm{~g}$, the pressure scale height is

$$
h(r)=0.5 \mathrm{au} \times\left(\frac{r}{r_{0}}\right)^{1.25} .
$$

Ignoring scattering and assuming that the disk is in local thermodynamic equilibrium (LTE), the emission from the disk is described by the radiative transfer equation

$$
\frac{d I_{\nu}}{d s}=K_{\nu}(s)\left(B_{\nu}(T)-I_{\nu}(s)\right),
$$

where $s$ is a spatial coordinate along the line of sight and $K_{\nu}(s)$ is the total opacity, $K_{\nu}(s)=\kappa_{\nu}(s) \rho(s)$. The assumption of LTE is valid, provided that the number density is much greater than the critical density of the gas. In the case of protoplanetary disks, this is reasonable, given that the critical density is given by

$$
n_{\text {crit }}=\frac{A_{i j}}{\langle\sigma \cdot v\rangle},
$$

where $A_{i j}$ is the Einstein $A$ coefficient, $\sigma$ is the collisional crosssection, and $v$ is the velocity. The angle brackets indicate and average over the velocity distribution. This is simply the ratio of the radiative decay rate, given by the Einstein $A$ value, to the rate coefficient for collisional de-excitation. The Einstein $A$ values for $\mathrm{CO}$ can be found in databases, such as the Leiden Atomic and Molecular Database. The rate coefficient for collisions between $\mathrm{CO}$ and various species, such as $\mathrm{H}_{2}$ and atomic hydrogen, can be found in databases such as the Basecol database (Dubernet et al. 2012). As an example, the Einstein $A$ value for the ${ }^{12} \mathrm{CO} \quad J=3-2$ transition is $6.910 \times 10^{-7} \mathrm{~s}^{-1}$, while the rate coefficient for collisions between ${ }^{12} \mathrm{CO}$ and $\mathrm{H}_{2}$ is $5.995 \times 10^{-11} \mathrm{~s}^{-1} \mathrm{~cm}^{-3}$, at $40 \mathrm{~K}$, although there is little variation with temperature. This gives a critical density of $1.15 \times 10^{4} \mathrm{~cm}^{-3}$. This is a small number density compared to those of protoplanetary disks. For example, a disk with mass $10^{-4} M_{\odot}$ and an outer radius of 100 au would have number densities in the midplane ranging from $10^{6}$ to $10^{8} \mathrm{~cm}^{-3}$. The density drops quickly with altitude, but even for this light disk model, the density is above critical for up to four scale heights at the inner radius and up to almost three at the outer radius. So, the critical density is smaller than the number density of the gas in all but the most remote and vacuous upper regions of the disk.

If the disk is viewed face-on, the radiative transfer equation can be integrated analytically, and the intensity as a function of 
the radius is (Appendix A)

$$
I_{\nu}(r)=B_{\nu}(T(r))\left(1-\exp \left(-k_{\nu}(r) \cdot \Sigma(r)\right)\right),
$$

where $\kappa_{\nu}$ is the mass absorption coefficient. Because we are in LTE, the source function is simply the Planck function. The optical depth of the disk emission is

$$
\tau_{\nu}(r)=\kappa_{\nu}(r) \cdot \Sigma(r) .
$$

Here, $\kappa_{\nu}$ includes all of the opacity sources. In the millimeter-wave regime, the disk opacity is dominated by dust and molecular gas, $k_{\nu}=\kappa_{\nu}^{d}+\kappa_{\nu}^{g}$.

Although the total opacity is simply the sum of the individual opacities, the dust and gas emission $\left(I_{\nu}^{d}, I_{\nu}^{g}\right)$ can only be separated in the optically thin case, where Equation (7) reduces to

$$
I_{\nu}(r)=\underbrace{B_{\nu}(T(r)) \kappa_{\nu}^{d}(r) \Sigma(r)}_{I_{\nu}^{d}}+\underbrace{B_{\nu}(T(r)) \kappa_{\nu}^{g}(r) \Sigma(r)}_{I_{\nu}^{g}} .
$$

If $\tau_{\nu} \geqslant 1$, the contributions from dust and gas each emit and attenuate each other and so cannot be simply separated. The implications of this fact in understanding molecular line observations will be discussed below.

In our model, we adopt dust opacities proper for spherical grains made of a mixture of silicates and carbonaceous material. The opacities are calculated following the procedure described in Isella et al. (2009). In brief, the optical properties of the different materials are combined using the Bruggeman theory with relative abundances as in Pollack et al. (1994). Opacities for dust grains with sizes $a$ between 0.01 and $1000 \mu \mathrm{m}$ are calculated using Mie theory and averaged over a grain size distribution $n(a) \propto a^{-3.5}$. The dust mass opacity at $0.87 \mathrm{~mm}$ is $1.44 \mathrm{~cm}^{2} \mathrm{~g}^{-1}$, which, for the disk model adopted here, gives an optical depth for the $0.87 \mathrm{~mm}$ dust emission of $\tau_{0.87 \mathrm{~mm}}(r) \sim 0.4 \times\left(r / r_{0}\right)^{-1}$.

Molecular gas absorbs and emits in lines, and its opacity depends on the temperature, density, and dynamics of the emitting material. In this section, we limit our analysis to the rotational transition $J=3-2$ of ${ }^{12} \mathrm{CO}\left(\nu_{0}=345.796 \mathrm{GHz}\right)$, the emission of which can be calculated assuming LTE. However, our results can be generalized to any line for which the LTE approximation holds true. The molecular opacity assumes the following form:

$$
\kappa_{i j}=\frac{c^{2}}{8 \pi \nu_{0}^{2}} \cdot \frac{g_{j}}{g_{i}} n_{i} \phi_{\nu} A_{j i}\left(1-\exp \left(-\frac{h \nu_{0}}{k T}\right)\right),
$$

where $\nu_{0}$ is the rest frequency of the transition, $g_{i}$ is the multiplicity of the $i$ th state, $n_{i}$ is the number density of ${ }^{12} \mathrm{CO}$ particles in the $i$ th state, $\phi_{\nu}$ is the line profile, $A_{i j}$ is the Einstein $A$ value, and $T$ is the temperature. For a detailed derivation, see Appendix B.

Figure 1 shows both the spatially integrated spectrum of the continuum and line emission, as well as their radial profile calculated at a frequency corresponding to the line center, for a disk mass of $2 \times 10^{-2} M_{\odot}$. In this simulation, we adopted a $\mathrm{H}_{2} /{ }^{12} \mathrm{CO}$ abundance ratio of $10^{5}$ and a $\mathrm{H}_{2} /$ dust ratio of $10^{2}$. The integrated spectrum of the emission has a characteristic boxy shape, with a flat top, due to the fact that the disk model is vertically isothermal and the central regions of the line in question are optically thick. Furthermore, because of the faceon inclination and because no turbulence is included in this model, the width of the line depends only on the thermal broadening (see Equations (27) and (28)). As discussed above, the continuum and line emission cannot be easily separated in Equation (7). However, we can calculate the pure line emission $I_{\nu}^{g}$, i.e., the line emission attenuated by dust absorption, by subtracting the pure continuum emission $I_{\nu}^{d}$, i.e., the emission of the continuum but attenuated by the line, from the total emission given by Equation (7). The pure continuum and line emission are therefore respectively defined as

$$
I_{\nu}^{d}(r)=I_{\nu}(r) \frac{k_{\nu}^{d}(r)}{k_{\nu}^{d}(r)+k_{\nu}^{g}(r)} \text { and } I_{\nu}^{g}(r)=I_{\nu}(r)-I_{\nu}^{d}(r) \text {. }
$$

Note that this assumes that the gas and dust are well-mixed. This is an approximation, as the dust settles over time and millimeter-sized dust grains will concentrate toward the midplane.

The optical depth of the line is shown in Figure 2, at line center. Although the temperature and density decrease monotonically with radius, the optical depth does not. This is because the opacity depends on the number density of $\mathrm{CO}$ molecules in the $J=3$ state, not just on the total number of molecules. In the inner disk, the temperature is very high, and the $\mathrm{CO}$ molecules equilibrate between a large number of levels, so the third level is less occupied. Farther out in the disk, the dropping temperature forces more molecules into the $J=3$ state. The maximum population in the $J=3$ state occurs at about $30 \mathrm{~K}$. On the other hand, past a certain point, the dropping temperature and total $\mathrm{CO}$ number density eventually becomes the dominant effect, and the optical depth decreases again. The peak occurs at around $35 \mathrm{au}$, where the line has an optical depth of $3.5 \times 10^{3}$, while the dust optical depth peaks at the inner radius, with a value of just 3 . CO molecules therefore absorb practically all of the continuum emission, causing it to drop by about three orders of magnitude compared to the continuum intensity outside the line. Similarly, at the outer radius of the disk, the optical depth of the line emission exceeds that of the continuum by five orders of magnitude. In practice, since the optical depth of the $\mathrm{CO}$ emission close to the line center is very large, no dust emission can escape from the disk. The part of the spectrum where the line emission has an optical depth greater than unity is indicated in the left panel of Figure 1, by the region between the two vertical lines. As discussed below, the absorption of continuum emission from $\mathrm{CO}$ molecules can have a large effect on the derivation of the gas temperature (and density) from the continuum-subtracted line intensity.

\subsection{Derivation of the Disk Temperature}

For thermal radiation, the intensity of the emission depends on the temperature of the emitting material. In radio astronomy, it is a common procedure to measure intensities in units of brightness temperature, defined as the temperature of a blackbody emitting the same power per unit of solid angle per unit of bandwidth. Intensities can therefore be converted into brightness temperature by inverting the Planck function:

$$
T_{B}=\frac{h \nu}{k_{B}}\left(\log \left(\frac{2 h \nu^{3}}{c^{2} I_{\nu}}\right)+1\right)^{-1} .
$$

If the emission is optically thick, the intensity depends only on the temperature of the emitting material $\left(I_{\nu}=B_{\nu}(T)\right.$; 

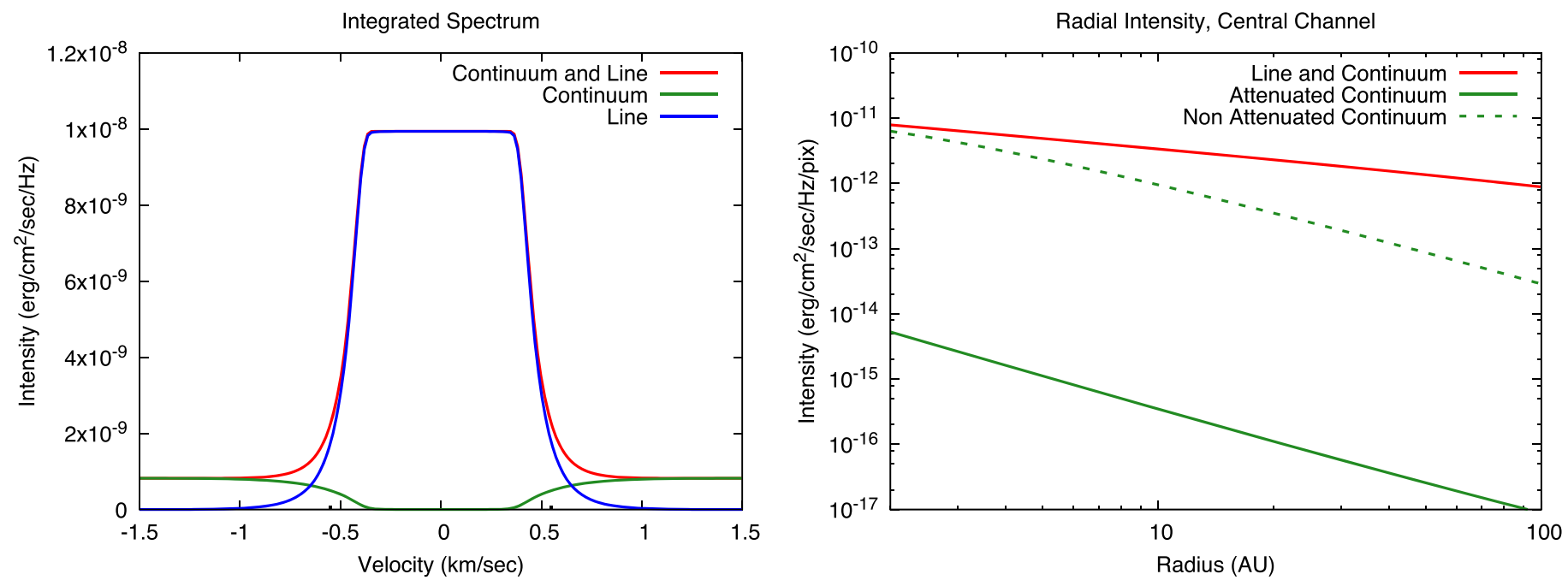

Figure 1. Left: spatially integrated spectrum of the ${ }^{12} \mathrm{CO} J=3-2$ line, for a disk of mass $2 \times 10^{-2} M_{\odot}$, showing emission from both line and continuum (red), pure line emission (blue), and the actual continuum emission accounting for absorption by the line (green). At line center, the continuum emission is almost zero. The vertical lines at roughly $0.6 \mathrm{~km} \mathrm{~s}^{-1}$ indicate the boundaries between the optically thin and optically thick portions of the spectrum. Even for this relatively massive disk, a fair fraction of the spectrum is primarily optically thin. The square profile arises from the assumption of a vertically isothermal temperature model, because all optically thick emission will have the same temperature. Right: intensity as a function of radius at line center, for the line and continuum (red), the attenuated continuum (green), and the continuum without line attenuation (dashed green). The attenuation lowers the continuum emission by three orders of magnitude.

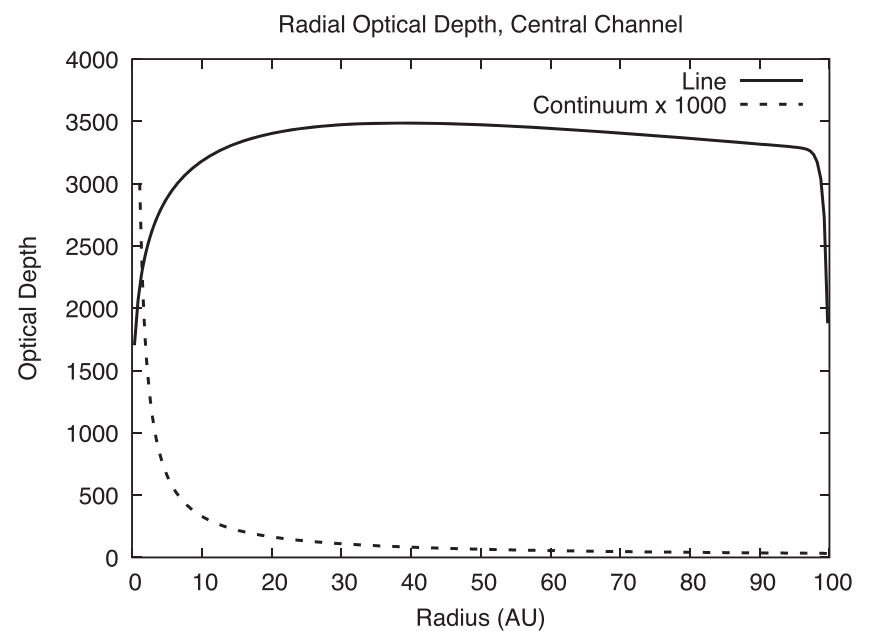

Figure 2. Optical depth of the ${ }^{12} \mathrm{CO} J=3-2$ line and the continuum as a function of radius, for a disk of mass $2 \times 10^{-2} M_{\odot}$. The optical depth of the line (solid line) peaks at around $35 \mathrm{au}$. Father out in the disk, it decreases because the density drops quickly. Closer in, the optical depth also drops, because rising gas temperature depopulates the $J=3$ level. The dashed line shows the optical depth of the continuum, magnified by a factor of 1000 , which behaves as a simple power law.

Equation (7)), and the brightness temperature is therefore equal to the physical temperature $\left(T_{B}=T\right)$. In general, the brightness temperature is related to the physical temperature by the relation

$$
B_{\nu}\left(T_{B}\right)=B_{\nu}(T)\left(1-e^{-\tau_{\nu}}\right),
$$

where $\tau_{\nu}$ is the optical depth of the emission (Equation (8)) ${ }^{1}$ In reality, however, a telescope like ALMA does not measure the intensity of the emission $I_{\nu}$ but its flux density $S_{\nu}$, which is the

\footnotetext{
1 In radio astronomy, it is common to define the brightness temperature assuming Rayleigh-Jeans approximations. In this case, Equation (13) simplifies to $T_{B}=T\left(1-e^{-\tau_{\nu}}\right)$. However, since the Rayleigh-Jeans approximations might not hold in the cold midplane regions of protoplanetary disks, we prefer to define the brightness temperature using the Planck function.
}

intensity integrated over the solid angle corresponding to the angular resolution of the telescope (see Condon \& Ransom 2016 for a comprehensive review of this topic). Flux densities are typically expressed in units of $\mathrm{Jy}_{\text {beam }}^{-1}$, where the beam is the FWHM of the synthesized clean beam. In the context of the synthetic models discussed in this section, the relevant solid angle to calculate the flux density is that subtended by the pixel size of our simulated images. The brightness temperature can still be defined using Equation (12), where the intensity is derived from the synthetic (or measured) flux density using the relation

$$
I_{\nu}=S_{\nu} / \theta^{2}
$$

where $\theta$ is the angular size of the pixels of the synthetic model (or the size of the synthesized beam in the case of real observations). This relation implies that the brightness temperature derived by our models (or by ALMA observations) is the average of the local brightness temperature calculated across the pixel (or the ALMA beam). As a consequence, if the emission of a thermal source is optically thick but arises from a region with angular area $A$ smaller than the area of the beam $\pi \theta^{2}$, its brightness temperature will be lower than its physical temperature by an amount equal to the ratio between the source size and the beam area. This effect takes the name of beam dilution and, as discussed in Section 4, has a direct impact on the observations of molecular line emission from protoplanetary disks. Conversely, if the emitting area is larger than the pixel (or beam), the measured brightness temperature is not affected by beam dilution. In summary, the relation between brightness temperature and physical temperature is

$$
B_{\nu}\left(T_{B}\right)= \begin{cases}B_{\nu}(T)\left(1-e^{-\tau_{\nu}}\right) \frac{\pi \theta^{2}}{A}, & \text { if } A<\pi \theta^{2} \\ B_{\nu}(T)\left(1-e^{-\tau_{\nu}}\right), & \text { if } A \geqslant \pi \theta^{2} .\end{cases}
$$

If the emission is optically thick and spatially resolved, as for example in the case of the ALMA observation of the CO line 
emission from nearby protoplanetary disks (see, for example, Schwarz et al. 2016), the exact knowledge of the optical depth is not necessary to derive the gas temperature. However, since the observers rarely know the exact optical depth, and because real disks are not vertically isothermal, the measured brightness temperature does not give the temperature at known points in the disk but rather describes a general region. Lines of differing optical depth can therefore be used to probe different regions of the disk to determine the vertical temperature structure. (See, for example, Dartois et al. 2003; Isella et al. 2007, or Rosenfeld et al. 2013). The case of nonvertically isothermal disks is discussed in Section 3.

\subsubsection{Effect of Continuum Subtraction}

The temperatures of protoplanetary disks can be determined from measuring the flux density of optically thick lines (if the area of the emitting source is known), but since an observation combines emission from both the line and the continuum, the contribution of the continuum must be quantified. The simplest and most common procedure is to assume that across the spectral width of the line, the continuum emission is approximately flat, and subtract it off. However, this procedure, commonly known as continuum subtraction, can lead to the removal of line emission if, as discussed in the previous section and shown in Figure 1, the line is optically thick and absorbs part or all of the underlying continuum. This effect is most severe in the denser regions of protoplanetary disks where both line and continuum emission tend to be optically thick. Standard continuum subtraction is accurate only in the optically thin limit represented by Equation (9).

The effects of continuum absorption on line emission are often considered in astrophysical applications, but here it is important to include the effects of line absorption on the continuum. Because an optically thick line can almost completely attenuate continuum emission, the emission at the peak of the line spectrum is entirely that of the line. The amount of line emission removed by assuming a flat continuum increases as the optical depth of the dust continuum emission increases, and it is maximized when both continuum and line emission are optically thick.

Because of this, the effect is not constant throughout the disk. The outer region has thinner dust and a lower continuum emission level. In the inner disk, however, the effect is particularly severe, as the dust is much brighter where both the temperature and the density are higher. In our vertically isothermal model, all optically thick emission will have the same magnitude, so if the dust reaches an optical depth greater than one, continuum subtraction will remove all emission. In more physically realistic temperature models, the effect may not be so severe as to remove all emission but may still lead to an erroneous estimate of the true line emission.

The (sub)millimeter-wave dust continuum emission has been found to be optically thick in several protoplanetary disks, including HL Tau, HD 163296, HD 142527, and IRS 48 (Casassus et al. 2013; Isella et al. 2016; Jin et al. 2016; van der Marel et al. 2016; Boehler et al. 2017). Integrated intensity maps of the continuum-subtracted molecular line emission from these sources show that the line emission is strongly suppressed where the dust continuum emission is more intense. In particular, inner cavities have been observed in the ${ }^{13} \mathrm{CO}$ and $\mathrm{C}^{18} \mathrm{O}$ emission toward the center of the HD 163296 disk, where the dust continuum is optically thick.
Similarly, a lack of molecular line emission has been observed in the optically thick dust crescents around IRS 48 and HD 142527 (van der Plas et al. 2014). These features can be explained by line removal by continuum subtraction.

Figure 3 shows the effect of continuum subtraction on ${ }^{12} \mathrm{CO}$ $J=3-2$ arising from disks with masses of $2 \times 10^{-2}$, $2 \times 10^{-3}, 2 \times 10^{-4}$, and $2 \times 10^{-5} M_{\odot}$. This range covers the range of masses of nearby protoplanetary disks. We adopted the pedagogical disk structure discussed above and calculated the brightness temperature $T_{B}$ of the line both before and after continuum subtraction. Furthermore, for each case, we calculated the brightness temperature from both the spectrally integrated (Moment 0) map and the peak emission map. The integrated emission map is calculated by averaging over the full spectral extent of the line (typically around $25 \mathrm{~km} \mathrm{~s}^{-1}$ for the inclined disk models), while the peak emission is the emission recorded at the peak of the spectral line. Because the chosen line is mostly optically thick and the synthetic model is not affected by beam dilution, the brightness temperature of the line should be very close to the physical temperature $T$ of the disk, and the ratio $T_{B} / T$ should therefore be close to 1 . We find instead that the brightness temperature derived from the continuum-subtracted line emission can be much lower than the physical temperature. The effect of continuum subtraction is most evident in the most massive disk model, where the brightness temperature of continuumsubtracted lines (red and green curves) drops sharply to zero within about $10 \mathrm{au}$ from the center. In practice, both the peak emission and integrated intensity maps (red and green lines) show an artificial inner cavity for the heaviest model when the continuum is subtracted. Obviously, the brightness temperature derived from the integrated intensity is always lower than the physical temperature. This is because the integrated line emission includes optically thin contribution from the line wings. However, as will be explained in Section 4, there are definite advantages to the use of integrated emission maps as they are robust to the effects of both noise and beam dilution.

The effect of continuum subtraction diminishes with the intensity of the continuum, and therefore with the mass of the disk, and becomes negligible in disks with masses less than about $10^{-4} M_{\odot}$. At disk masses lower than $10^{-5} M_{\odot}$, the optical depth at the peak of the ${ }^{12} \mathrm{CO} 3-2$ line drops below 5, and the brightness temperature of the line is systematically lower than the physical disk temperature. Interestingly, the optical depth of the line has a minimum in the innermost (and densest) regions of the disk. This counterintuitive behavior is because the level populations of the $\mathrm{CO}$ rotational modes depend on the temperature, and in the inner disk, the very high temperature depopulates the third level. This leads to a lower optical depth for the 3-2 line, despite the higher gas density.

For weaker lines, where the optical depth of the line is not significantly greater than that of the dust, non-continuumsubtracted peak emission maps will still offer better results than continuum-subtracted integrated emission maps, but the brightness temperature will still fall short of the physical temperature if the optical depth is low. Nonetheless, for all models, the noncontinuum-subtracted peak emission map (orange line) gives the closest result to the physical temperature.

\subsection{Effect of Gas to Dust Ratio}

Previous discussion has focused on models where the gas to dust ratio is 100 , roughly the value of the nearby interstellar 

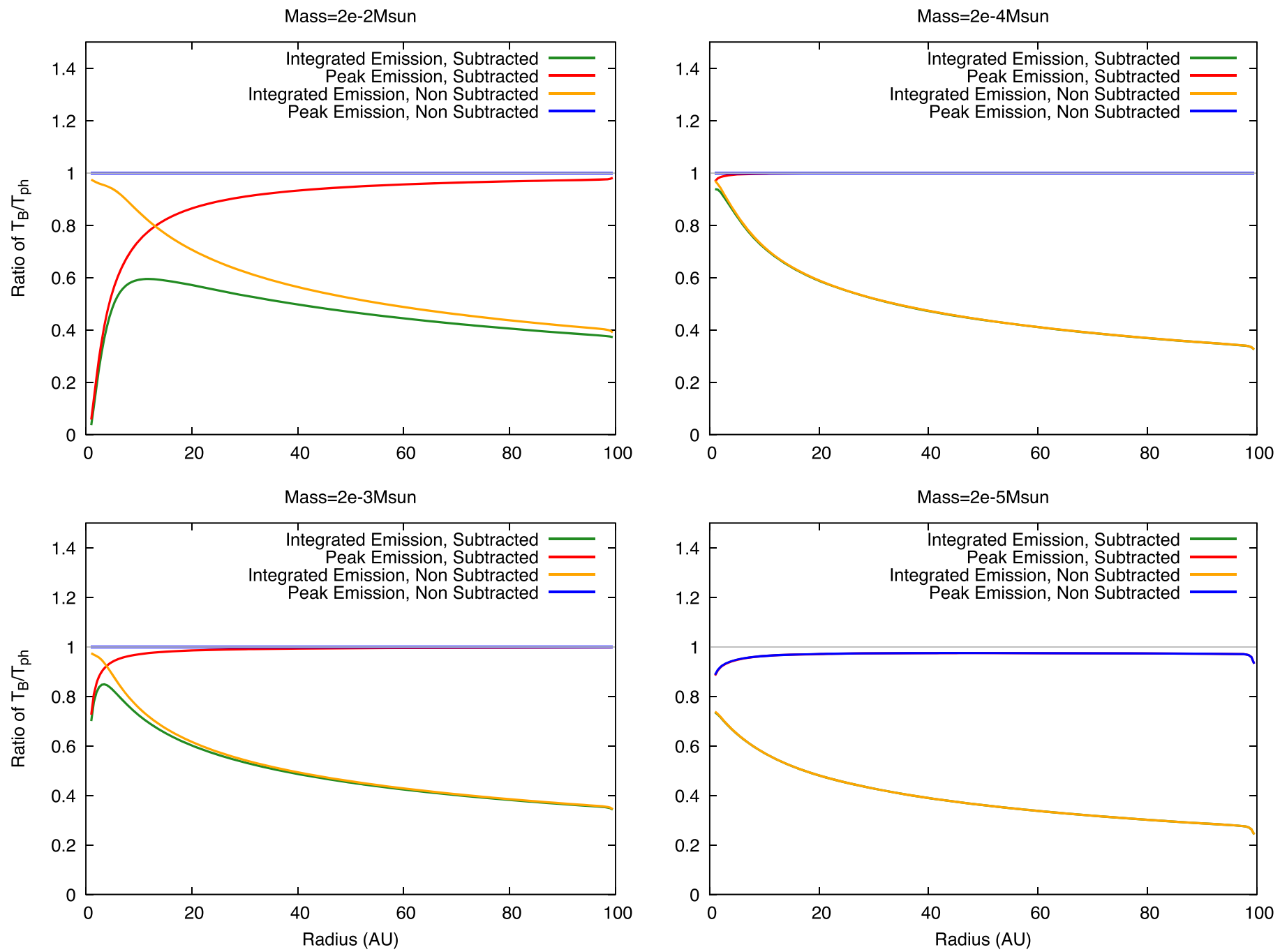

Figure 3. Ratios of brightness temperature to physical temperature for a variety of different methods and models. Each panel corresponds to a different disk mass, ranging from $2 \times 10^{-2} M_{\odot}$ to $2 \times 10^{-5} M_{\odot}$. Within each panel, the four lines correspond to the integrated emission map and peak emission map methods, both with and without continuum subtraction.

medium. However, it is likely that the ratio is not constant across all disks, or even across a given disk. (See, for example, van der Marel et al. 2016; Boehler et al. 2017, where the crescent visible in HD 142527 and IRS 48 is found to contain far more dust than other regions of the disk.)

The effects of an altered gas to dust ratio are as follows: in cases where the gas to dust ratio is lower than the ISM value, the effects of continuum subtraction will be increased, while the effects of optically thin emission being included will not change. Lowering the gas to dust ratio increases the amount of dust present, which means that the background level of emission away from the line will increase. Thus, there is more emission that can be incorrectly attributed to the continuum if the continuum is subtracted. However, until the continuum emission is high enough to be optically thick, the range of frequencies being integrated over will contain roughly the same fraction of optically thin and thick emission.

Figure 4 shows the effect of assuming a gas to dust ratio of 10 (instead of 100) on the derivation of the disk temperature. While Figure 4 is qualitatively similar to Figure 3, the effects of continuum subtraction are more exaggerated, particularly in the inner region of the disk. In the outer disk, deviation from the best measurable temperature is given primarily by the inclusion of optically thin emission, and so does not show much change from the different gas to dust ratio.

The relation between disk physical temperature and line brightness temperature depends on the line optical depth, and therefore, on the abundance of the emitting molecules. ${ }^{12} \mathrm{CO}$ is an abundant molecule in disks and its low- $J$ transitions are optically thick even for relatively low $\mathrm{CO}$ column densities. $\mathrm{CO}$ isotopologues such as ${ }^{13} \mathrm{CO}$ and $\mathrm{C}^{18} \mathrm{O}$ are less abundant but still easily detected. If the disk is massive, even the line emission from $\mathrm{CO}$ isotopologues can be optically thick and allow the disk temperature to be probed. $\mathrm{C}^{18} \mathrm{O}$, being more likely to be optically thin, can be used to determine gas masses. (See Williams \& Best 2014.)

Figure 5 shows the ability for the temperature of various disk masses for the cases of ${ }^{12} \mathrm{CO},{ }^{13} \mathrm{CO}$, and $\mathrm{C}^{18} \mathrm{O}$ to be recovered. All temperatures are measured via the non-continuum-subtracted peak emission map, since that technique has already been demonstrated to work best for disks with emission becoming optically thin. Because ${ }^{13} \mathrm{CO}$ and $\mathrm{C}^{18} \mathrm{O}$ are much less abundant than ${ }^{12} \mathrm{CO}$, which limits their utility as temperature tracers, they offer consistently less useful results. ${ }^{12} \mathrm{CO}$ is optically thick at its peak for all disk masses covered here, except for the lightest model, and even in that case, the emission is still quite close to 

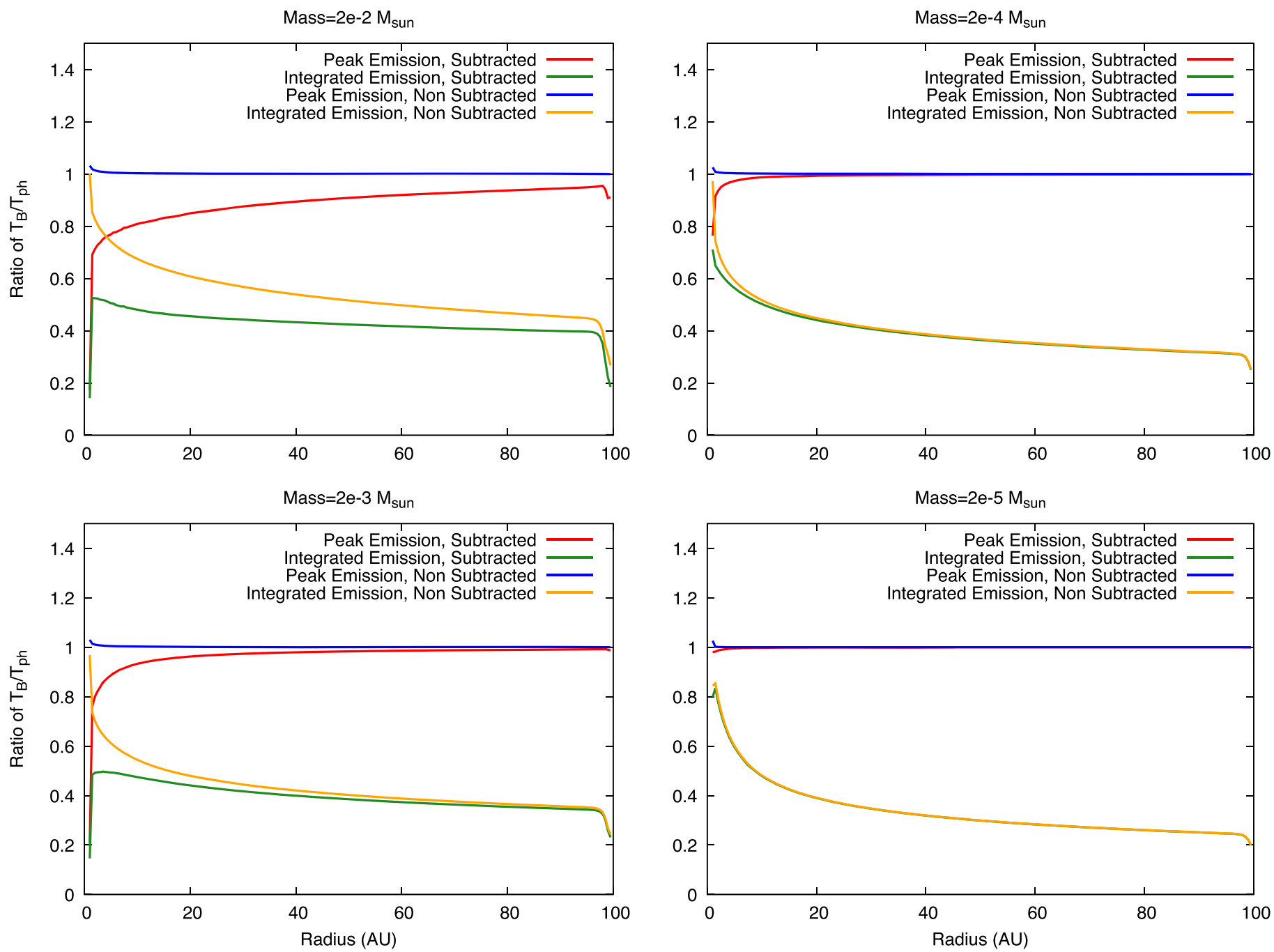

Figure 4. Relative accuracies of the radial temperature for different masses of the disk model for the integrated and peak emission maps, for a variety of disk masses. For these models, the gas to dust ratio is much higher than the ISM value, set to 10 instead of 100 . As expected, the effects of continuum subtraction are now more severe than before, especially for high-mass models.

being optically thick. ${ }^{13} \mathrm{CO}$ is abundant enough to be useful for the heavier disk models, but by $2 \times 10^{-4} M_{\odot}$, the emission is too thin to be useful. $\mathrm{C}^{18} \mathrm{O}$ is uncommon enough that it can only be used for the heaviest of disks.

An effect similar to that of an altered gas to dust ratio is the freezing out of $\mathrm{CO}$ molecules onto dust grains at low temperature. For $\mathrm{CO}$, freeze-out typically takes place at temperatures of around $20 \mathrm{~K}$ (see Öberg et al. 2011). This is a fairly low temperature but is still reached by a fair fraction of the disk midplane. (See Figure 6, where the white line denotes the $20 \mathrm{~K}$ contour.) The implications are as follows: for more abundant isotopologues, such as ${ }^{12} \mathrm{CO}$, there will be no noticeable effect, because the line becomes optically thick high enough in the disk atmosphere that the freeze-out in the midplane is irrelevant. For less abundant isotopologues, such as $\mathrm{C}^{18} \mathrm{O}$, where the line becomes optically thick nearer the midplane (if it does at all), there will be a substantial loss of flux due to freeze-out. The primary emitting area, at the midplane, where density is highest, is depleted, and so the line is much less optically thick.

Because freeze-out simply removes molecules that would have emitted, it has the same effect as lowering the disk mass (or raising the gas to dust ratio). However, because of the temperature dependence, the effect is only significant for less common isotopologues. The primary hazard for observers regarding freeze-out is that a line that might be assumed to be optically thick based on the disk mass or brightness of the ${ }^{12} \mathrm{CO}$ line may not be because all of the $\mathrm{CO}$ at the midplane is frozen out.

There are additional subtle effects that can also alter the abundances of $\mathrm{CO}$ and its isotopologues. Possible depletion can come from active disk chemistry (see Kama et al. 2016). Another such effect is CO self-shielding (see Schwarz et al. 2016).

\section{Physical Disk Model}

\subsection{Structure and Emission}

The models discussed above contain all of the relevant features of protoplanetary disks but are nonetheless extremely simple models, particularly regarding the vertical temperature profile. A more realistic temperature profile for the outer regions of an externally illuminated disk would have a colder midplane and hotter disk surface, assuming that viscous heating is negligible (see Dullemond et al. 2002). Such a temperature structure can be obtained by solving the radiative transfer 
${ }^{12} \mathrm{CO}$

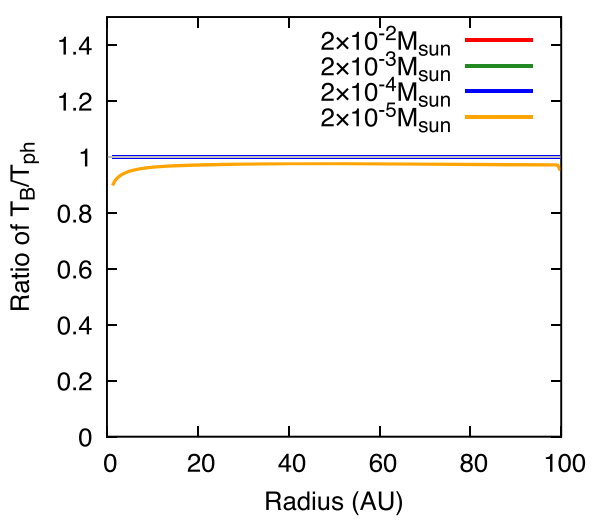

${ }^{13} \mathrm{CO}$

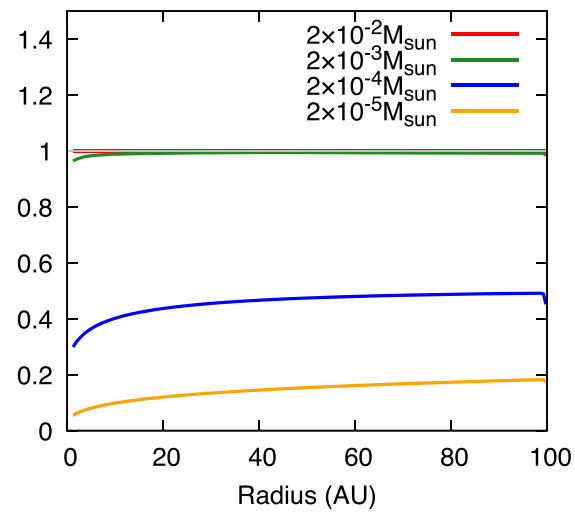

$\mathrm{C}^{18} \mathrm{O}$

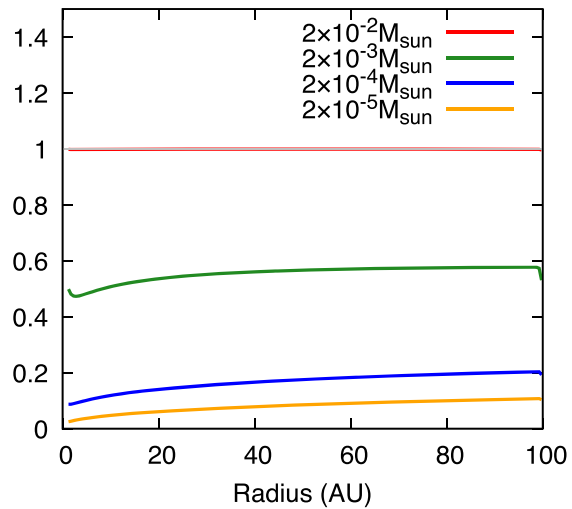

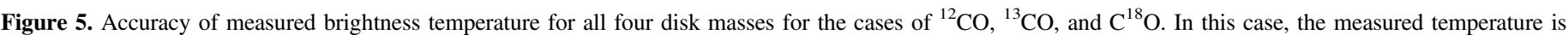

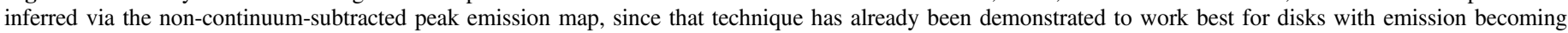
optically thin.

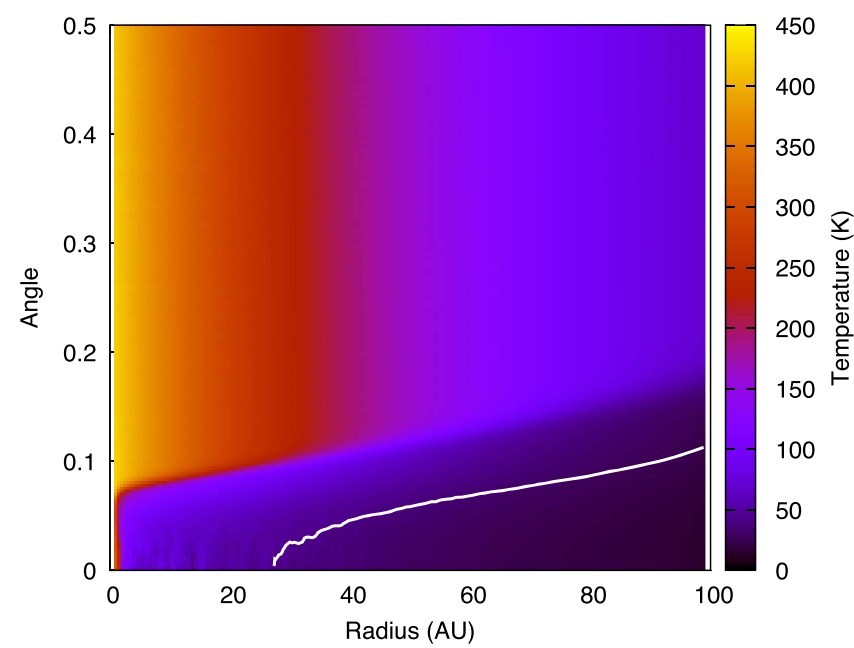

Figure 6. Example temperature profile for a disk of mass $2 \times 10^{-2} M_{\odot}$, as calculated using RADMC-3D. The angle is measured relative to the midplane. The radial temperature profile is similar to previous models, but now the vertical temperature gradient varies, starting at the very cold midplanar regions and heating up at the lower density, high altitudes. The temperature profile is symmetric about the midplane. Separate temperature models were generated for each disk mass; however, they are qualitatively highly similar. The white line indicates the $20 \mathrm{~K}$ contour. Inside this region, $\mathrm{CO}$ freezes out onto dust grains.

equations taking into account all of the heating and cooling sources as well the vertical disk structure. Here, we use the Monte Carlo radiative transfer code RADMC-3D (Dullemond 2012) to calculate the temperature distributions $T(r, z)$ of disks characterized by the same surface densities and masses discussed in the previous section. Figure 6 shows an example temperature profile for a disk of mass $2 \times 10^{-2} M_{\odot}$. However, it is worth mentioning that high in the disk atmosphere, the temperature of gas and dust may no longer match. When $n<n_{\text {crit }}$ (see Equation (6)), the gas is no longer in LTE and can be much hotter than the dust (see Bergin et al. 2016).

Because of the vertical temperature profile, it is no longer possible to determine the dust and gas intensity analytically, and it is also no longer possible to simply describe the ratio between the line brightness temperature and the physical disk temperature as done in the previous section. However, we can use ray-tracing calculations to investigate the accuracy of the inferred gas temperature from the line emission. To this end, we created our own ray-tracing algorithm, which allows us to numerically calculate the pure line and continuum emission. This algorithm differs from the ray-tracing module of RADMC-3D in that it numerically integrates the line and continuum radiative transfer equation (Equation (5)) without the need to specify a three-dimensional grid, and it is written to use parallelization to speed up the ray tracing. The details of the ray-tracing code and a comparison with RADMC-3D are presented in Appendix C.

In the preceding sections, we established that the noncontinuum-subtracted peak emission of an optically thick line is the best probe of the temperature of the emitting gas. However, if the disk is not vertically isothermal and/or not observed at a face-on inclination, the brightness temperature of the line would correspond to an average temperature over the region where the line becomes optically thick rather than to a specific point. It is therefore important to consider the morphology of the emitting region. Figure 7 shows the location of the disk regions emitting ${ }^{12} \mathrm{CO} J=3-2$ as observed at a face-on inclination. The lightly shaded region of each panel represents the region responsible for $90 \%$ of the line emission, while the more heavily shaded area corresponds to the region producing the inner $33 \%$ of the line emission. The line represents the height at which the observed brightness temperature matches the physical temperature of the disk.

The position where the brightness temperature matches the physical temperature of the gas for ${ }^{12} \mathrm{CO}$ depends on the optical depth of the line, and therefore, on the disk mass. For disks more massive than $M=2 \times 10^{-3} M_{\odot}$, the point where the brightness temperature is equal to the physical temperature of the gas is located between two and three scale heights. At lower masses, the location moves toward the midplane. The height of the emitting layer increases from about $1 h$ for $M=2 \times$ $10^{-5} M_{\odot}$ to $3 h$ for $M=2 \times 10^{-2} M_{\odot}$. Consequently, the temperature of the line will decrease with the disk mass from a value similar to that of the disk surface, which is typically located between four and five scale heights (see, e.g., Dullemond et al. 2001), to that of the disk midplane.

The point of matching temperature is roughly in the middle of the emission region, as expected. The width of the emission region has important implications for determining vertical temperature gradients for protoplanetary disks. At around a scale height, the emission region is thick and represents a fair fraction of the disk area. Because of this, lines of lower optical 

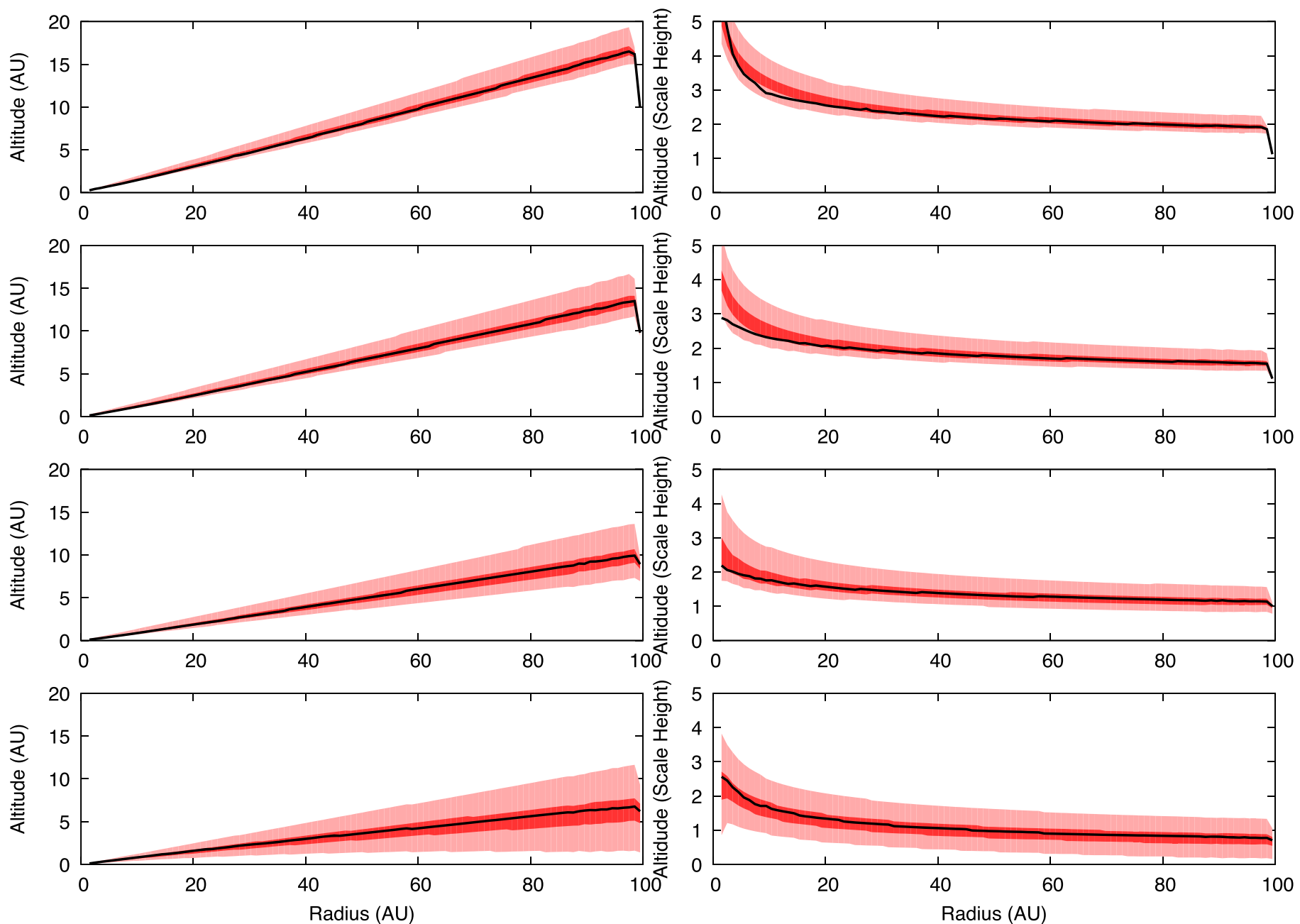

Figure 7. Regions dominating disk emission for face-on disk models with masses from $2 \times 10^{-2} M_{\odot}$ to $2 \times 10^{-5} M_{\odot}$. The left column shows the emission region in the RZ plane. The right column displays the same data, but with the altitude in terms of scale heights, not astronomical units. The lightly shaded region corresponds to the region producing $90 \%$ of the emission, while the darker shaded region corresponds to the inner $33 \%$ of the emission. The line represents the height at which the observed brightness temperature matches the physical temperature of the disk.

depth can overlap, complicating the derivation of the vertical temperature structure.

\subsection{Derivation of Gas Temperature from the Line Emission}

Following the analysis presented in the previous session, we first decompose the line emission in true gas emission, i.e., the line emission attenuated by dust absorption, and true dust emission, i.e., the continuum emission attenuated by gas absorption. Due to the vertical temperature gradients, Equation (11) no longer holds, and these two terms must be calculated numerically. We do that by performing two raytracing simulations: the first includes both gas and dust opacities to calculate the combined emission. In the second, we only use the dust opacity in the source function term and both dust and gas opacities in the attenuation term of the radiative transfer equation. These two emissions are then subtracted to obtain the true gas emission, which is finally converted to a true brightness temperature using Equation (15). If the line is optically thick, the true line brightness temperature will correspond to a weighted average of the physical gas temperature across the region emitting the line (see Figure 7). Instead, if the line is optically thin, the true line brightness temperature will be lower than the temperature of the emitting layer.

As before, we use the ratio between the brightness temperature of the line derived from both peak and integrated emission, both with and without continuum subtraction, and the true brightness temperature to investigate the most reliable method for deriving gas temperatures. Figure 8 shows this ratio as a function of radius for the same disk models shown in Figure 3. The difference is that the disks are no longer vertically isothermal. However, as in the vertically isothermal case, the non-continuum-subtracted peak emission map is the best probe of gas temperatures, while continuum subtraction leads to the gas temperature in the regions where the continuum is optically thick to be underestimated. This effect is less severe than in the isothermal case, because the disk midplane, which is where most of the dust emission originates, is colder than the layer emitting the line. The brightness temperatures derived from the continuum-subtracted integrated intensity (yellow lines) are, on average, half of the gas temperature and should therefore not be used for this purpose.

It is worth noting one crucial difference between vertically isothermal and non-isothermal models. The temperature derived from the non-continuum-subtracted peak intensity in 

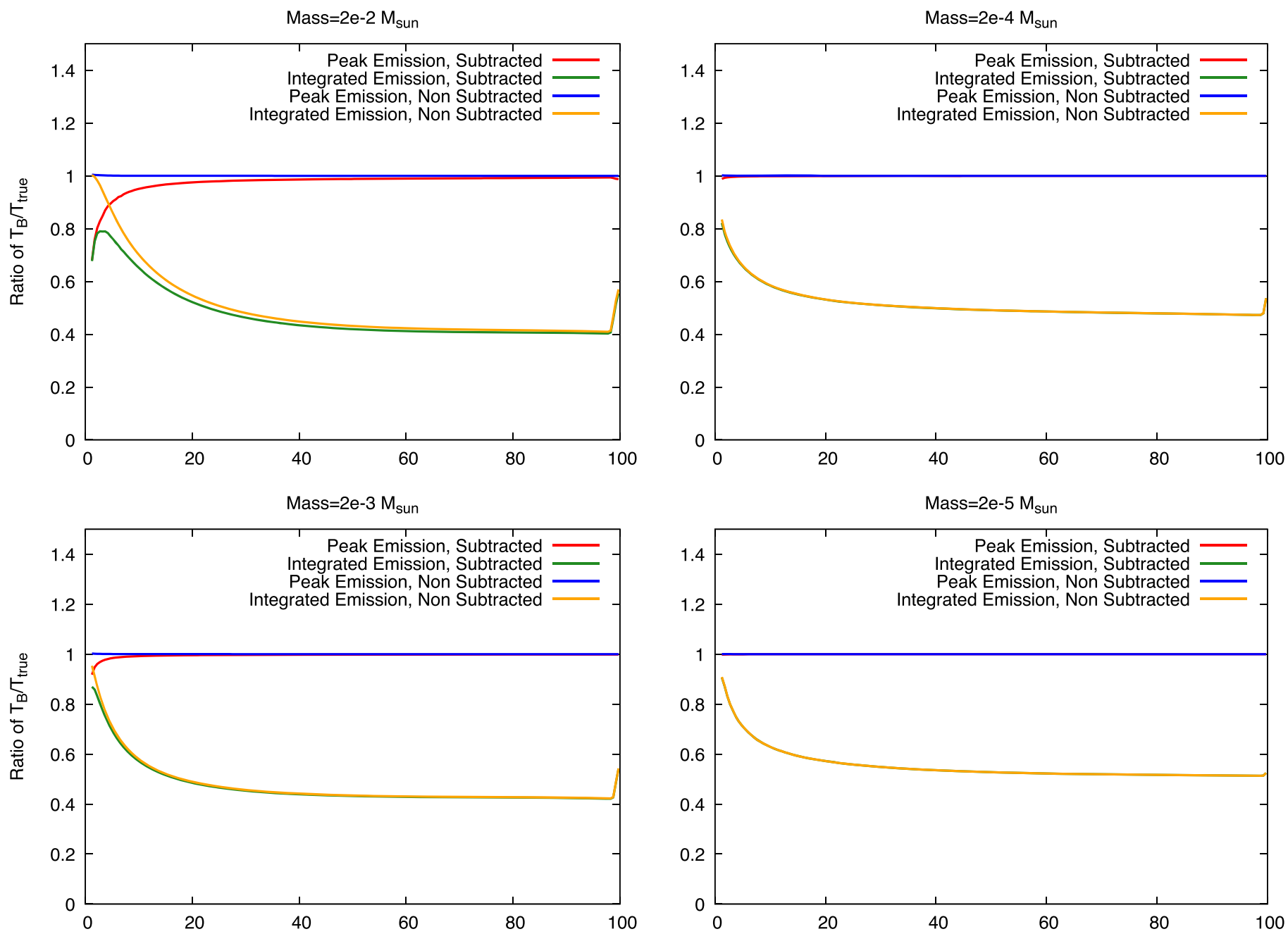

Figure 8. Relative accuracies of radial temperature for different masses of the disk model for the integrated emission map and for the peak emission map. In these models, the disk is no longer vertically isothermal. Since there is no longer a single physical temperature to recover at each radius, the comparison is against the most accurate possible brightness temperature that can be recovered.

the non-isothermal model appears to trace the gas temperature much better than in the isothermal model, particularly at low mass and high radius. This is a result of the fact that while in Figure 3 we compare the line brightness temperature to the real gas temperatures, in Figure 8 we no longer can, because the model is not vertically isothermal, and no single temperature characterizes a given radius. Instead, we compare the line brightness temperature with the true brightness temperature calculated by subtracting the attenuated continuum model from the full model. In the case of optically thick emission, the true brightness temperature is equal to the physical temperature of the emitting region, but for the lower mass and more optically thin models, both the true brightness temperature and the brightness temperature inferred from the observations will be lower than the gas temperature.

In the disk models presented here, the optical depth of the ${ }^{12} \mathrm{CO} 3-2$ line emission at the center of the line is about $3.6 \times 10^{4}$; at the inner radius of the most massive disk, it is about 10 at $60 \mathrm{au}$ in the $2 \times 10^{-5} M_{\odot}$ disk model, and never quite reaches unity, even for the lightest model. This comes primarily from ${ }^{12} \mathrm{CO}$ being a very abundant molecule, and the $3-2$ line being quite bright at these temperatures. This means that the line emission remains optically thick $(\tau>1)$ for the peak of the line even at CO column densities of $4.2 \times 10^{-7} \mathrm{~g} \mathrm{~cm}^{-2}$ or number column densities less then $1.8 \times 10^{16} \mathrm{~cm}^{-2}$. The location at which the line brightness temperature inferred from the non-continuum-subtracted peak emission is equal to the gas temperature is indicated by black curves in Figure 7 . When the line is optically thick, the brightness temperature of the line probes the temperature of a disk layer included in the region that emits most of the line. As the line becomes optically thin, the brightness temperature decreases until it drops below the temperature of the gas on the disk midplane, which is the lowest gas temperature value in each vertical column. In the face-on case, measurements of optically thick emission lines characterized by different optical depths could therefore allow the three-dimensional disk vertical structure if the optical depth of the line is known to be estimated.

\subsection{Effect of Inclination}

For ease of analysis, the preceding discussion has considered only disks which are viewed face on. However, most actual observations must deal with some degree of inclination. The primary effects of tilting the disk are as follows. First, Keplerian rotation will introduce a velocity component along the line of sight, with the result that for a given frequency window, only a small region will be emitting strongly. Because 
of this, the morphology of the spectrum changes significantly. Instead of being a relatively simple, singly peaked distribution, the spectrum becomes much wider and might show two peaks symmetric with respect to the center of the line. In terms of measuring a brightness temperature, however, there is little change from the face-on case. The spectrum for an inclined disk is more spread out in frequency than the sharply peaked spectra from face-on disks and has larger contributions from optically thin regions. This implies that temperatures derived from the spectrally integrated measurement will underestimate the gas temperature by an even larger amount compared to the face-on case. Therefore, in order to more concisely discuss the effect of the disk inclination on the determination of the gas temperature, in this section we will restrict the analysis to the line peak emission.

Because of the non-face-on inclination, the disk emission is no longer azimuthally symmetric, and we can no longer plot temperatures as a function of the orbital radius. Instead, we show in Figure 9 two-dimensional images of the ratio between the true brightness temperature and the line brightness temperature derived from peak intensity maps. The models correspond to disk masses of $2 \times 10^{-2} M_{\odot}, 2 \times 10^{-3} M_{\odot}$, and $2 \times 10^{-4} M_{\odot}$, observed at an inclination of $60^{\circ}$.

Without continuum subtraction, the line brightness temperature is slightly higher than the true brightness temperature closer to the center of the disk and along the disk outer edge. This is because tilting the disk increases the length of the lines of sight through the disk, causing the optical depth of both gas and continuum emission to increase. This effect increases as the inclination increases, and it is most noticeable in the heaviest model with mass $2 \times 10^{-2} M_{\odot}$, as the effect is most pronounced where dust emission is more significant. Lighter models are largely unaffected, because the disk is so light that even in the denser inner regions the dust emission is so negligible that there simply is nothing to incorrectly attribute to the line. In any case, including the continuum emission in estimating the line temperature leads to errors smaller than $5 \%$.

If the continuum is subtracted, as shown in the left column of Figure 9, the error goes in the opposite direction, and the emission is underestimated. The underestimate from continuum subtraction is larger than the overestimate obtained from not subtracting the continuum and can be as high as $10 \%-15 \%$ in the most massive case. Recall, however, that the apparent accuracy in this case is only relative to the true brightness temperature, and that for optically thin emission, the brightness temperature is far below the gas temperature.

This example considered a disk inclined by $60^{\circ}$. Because the disks are geometrically thin, the lines of sight will lengthen as the disk is inclined. This means that the effects described above will be magnified for higher inclinations.

Finally, it is important to consider the emission region of the inclined disk, as was done for the face-on models. In the more geometrically complicated inclined case, the lines of sight are no longer straight down, and the results are not radially symmetric. Additionally, the Keplerian rotation of the disk means that only a fraction of the gas is emitting at the right frequency.

Figure 10 shows two maps of the emission region for the inclined disk of mass $2 \times 10^{-3} M_{\odot}$, for the ${ }^{12} \mathrm{CO} \mathrm{3-2} \mathrm{line,}$ imaged at $1 \mathrm{~km} \mathrm{~s}^{-1}$ off of line center. The left panel shows the height of the emission region (defined as the height above the midplane of the centroid of each line of sight) divided by the radius. The right panel shows the width of the emission region, defined as the region producing the middle $90 \%$ of the emission, divided by the height of the center. Because of the Keplerian rotation of the gas, most of the gas picks up a line-ofsight velocity, and the line emission is Doppler-shifted out of the frequency window. Only a small region still emits at the correct frequency. This region is dominated by $\mathrm{CO}$ emission, which is optically thick, and the emission region behaves similarly to that in Figure 7 . The center of the emission region is located above the midplane, because the $\mathrm{CO}$ emission quickly becomes optically thick. The width of the emission region is small, roughly $30 \%-40 \%$ of the width away from the center, because the optical depth rises quickly. As the line weakens, away from the $y$ axis, the width increases as the optical depth drops. The width also increases farther away from the center, for the same reason. As the disk mass increases, the emission region shifts to higher altitude and becomes narrower.

\section{Derivations of the Gas Temperature from Synthetic Observations of a Physical Disk Model}

Lastly, it is key to consider the effects that a real observation would have on recovering an accurate line brightness temperature and, ultimately, the gas temperature. The discussion thus far has focused on synthetic disk models, and has ignored the fact that interferometric observations are affected by finite noise and resolution as well as spatial filtering. In this section, we first investigate the effect of the finite angular resolution by convolving synthetic disk models of the $\mathrm{CO}$ emission with two-dimensional Gaussian functions that mimic ALMA point spread functions. We then investigate the combined effect of noise, angular resolution, and spatial filtering by producing synthetic ALMA observations of our disk models.

\subsection{Effect of Beam Convolution}

We first investigate the effect of beam convolution on the emission from the pedagogical disk model discussed in Section 2. Though not realistic, this model has the advantage of allowing us a precise comparison between the line brightness temperature $T_{B}$ and gas temperature $T_{\mathrm{ph}}$. Figure 11 shows the radial profile of the ratio $T_{B} / T_{\mathrm{ph}}$ corresponding to the $\mathrm{CO}$ emission from the $2 \times 10^{-3} M_{\odot}$ disk model convolved with Gaussian beams of 0 ! $16 \times 0$ ". 14 and 0 " $11 \times 0$ ". 09 (top and bottom panels, respectively). This case is analogous to the lower-left panel of Figure 3. Convolution with the beam smears out emission and has the most effect where the intensity changes rapidly. Thus, the inner and outer edges of the disk are strongly affected, and the ability to determine the correct brightness temperature is reduced. Beam dilution is the strongest within half a beam from the center, and therefore it becomes more severe as the beam size gets larger. The problem of beam dilution is independent of the choice of peak emission map or integrated emission map and the choice of continuum subtraction. All methods are affected equally.

To further demonstrate the effects of beam convolution, we present in Figure 12 synthetic brightness temperature maps of the ${ }^{12} \mathrm{CO} J=3-2$ emission from the $2 \times 10^{-3} M_{\odot}$ nonvertically isothermal disk model observed at an inclination of $30^{\circ}$. The maps correspond to the line plus continuum emission calculated at a velocity of $1 \mathrm{~km} \mathrm{~s}^{-1}$ and show the effect of the 

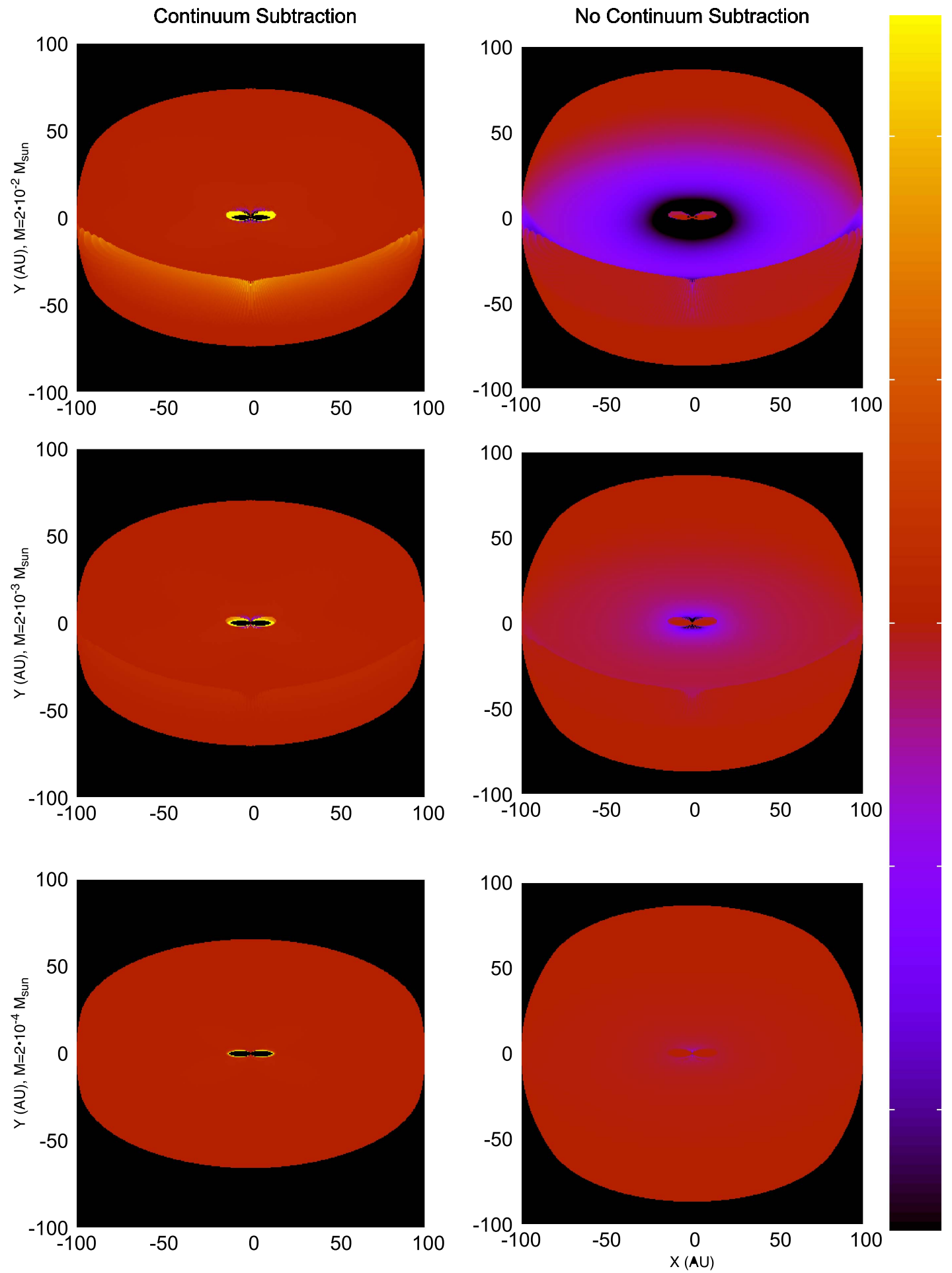

Figure 9. Accuracy of brightness temperature measured via the peak emission map for masses ranging from $2 \times 10^{-2} M_{\odot}$ to $2 \times 10^{-4} M_{\odot}$, with and without continuum subtraction, for an inclination of $60^{\circ}$. Deviation from the best recoverable brightness temperature is greatest toward the center and along the surface where the tilted disk most increases the line of sight. The deviation is only of order a few per cent.

convolution with beams of 0 !" $11 \times 0$ ". 09 and 0 "! $16 \times 0$ "! 14 in size.

Due to the effect of beam dilution, the brightness temperature of the line decreases with increasing beam sizes. This effect is strongest where the emitting area is smallest, as in the central region of the disk where, due to Keplerian rotation, the emitting region in a given channel becomes extremely narrow. This means that very small beam sizes are required to measure gas temperatures in the innermost disk regions. In the case of a beam size of 0 "! $11 \times 0 . \prime 09$, the brightness temperature of the line reaches a maximum of $67.3 \mathrm{~K}$, while the true brightness temperature in the same pixel is $73.7 \mathrm{~K}$. However, in the more strongly affected central disk region of the channel map, the true brightness temperature reaches a maximum of $159.1 \mathrm{~K}$, but the beam-convolved model only has a value of $23 \mathrm{~K}$. This implies that beam dilution has a strong effect on measuring gas 

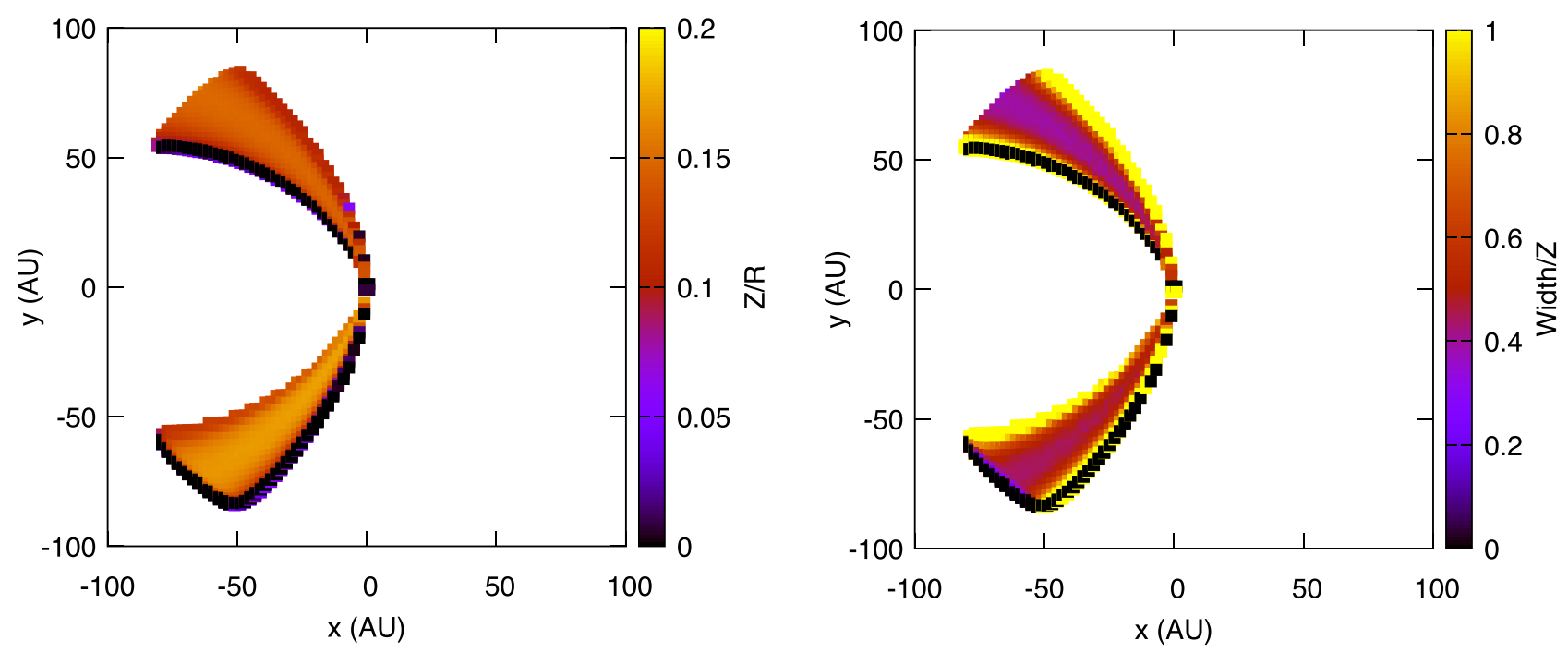

Figure 10. Emission region of a disk inclined at an angle of $60^{\circ}$, for a mass of $2 \times 10^{-3} M_{\odot}$. The emission comes only from the ${ }^{12} \mathrm{CO} 3-2$ line, measured $1 \mathrm{~km} \mathrm{~s}{ }^{-1}$ off of line center. Left: each point represents the height of the center of the emission region for that point in the disk, shown as $Z / R$. Right: the width of the emission region, divided by the height of the center at each point. At points of high optical depth, the center of the emission region is higher above the disk midplane, and the region is narrower.
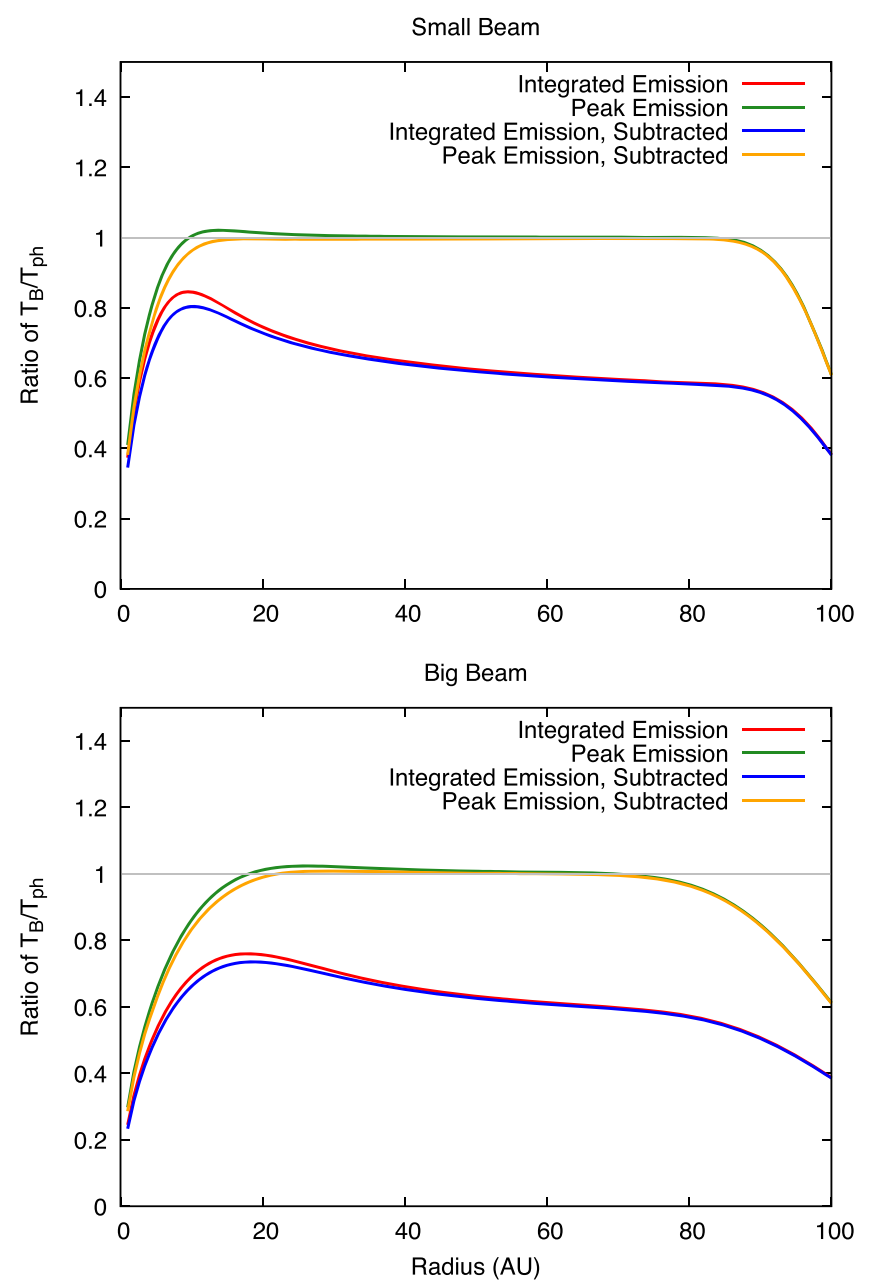

Figure 11. Ratios of measured brightness temperature to physical temperature for a vertically isothermal, face-on disk with a mass of $2 \times 10^{3} M_{\odot}$. Top panel: models convolved with a 0 ! $11 \times 0$." 09 beam. Bottom panel: models convolved with a 0 ! $16 \times 0$ !. 14 beam. In both cases, beam dilution primarily causes problems in the inner- and outermost regions of the disk.

temperatures in protoplanetary disks, even at the resolutions provided by ALMA. Clearly, ALMA is capable of even higher resolution. However, since the integration time required to achieve a given signal-to-noise ratio increases as $\theta^{-4}$, observations of the line emission at a resolution smaller than about $0 . " 1$ requires a considerably large amount of telescope time.

Beam dilution affects the integrated emission map and peak emission map differently. In particular, our simulations show that the spectrally integrated emission map is strongly resilient against the effects of beam dilution. The reason for this is that although the beam convolution smears out the emission, the end result is that flux lost from one resolution element is moved in the nearby resolution element. As a consequence, beam dilution leads to a spectral broadening of the line such that despite the fact that the peak emission might substantially drop, the integral is roughly the same.

This conclusion is supported by Figure 13, which shows histograms of the ratio between the line brightness temperature derived both from integrated emission and peak emission maps, and the true line brightness temperature for the disk model discussed above convolved with beam sizes of 0 " $11 \times 0$ " 09 and 0 " $16 \times 0$ " 14 . In both cases, the curves corresponding to the temperature derived from integrated emission maps show a strong peak around 1 , with $90 \%$ of the values having ratios between 0.73 and 1.31 for the larger beam, and 0.77 and 1.27 for the smaller beam. Temperatures derived from the peak emission map are instead spread across a large range of values. This is because most of the inner region of the disk has lost flux because of beam dilution, and the recovered brightness temperature is low by an average of $10 \%-20 \%$, but with some pixels being much worse. This demonstrates the primary constraint on the use of the peak emission map method: the beam size must be small compared to the size of the channel maps in order for the results to be worthwhile. The disk considered in Figure 13 is only 100 au in radius, making it fairly small. Figure 14 instead shows the effects of observing a larger disk model, where the radius has been increased to 

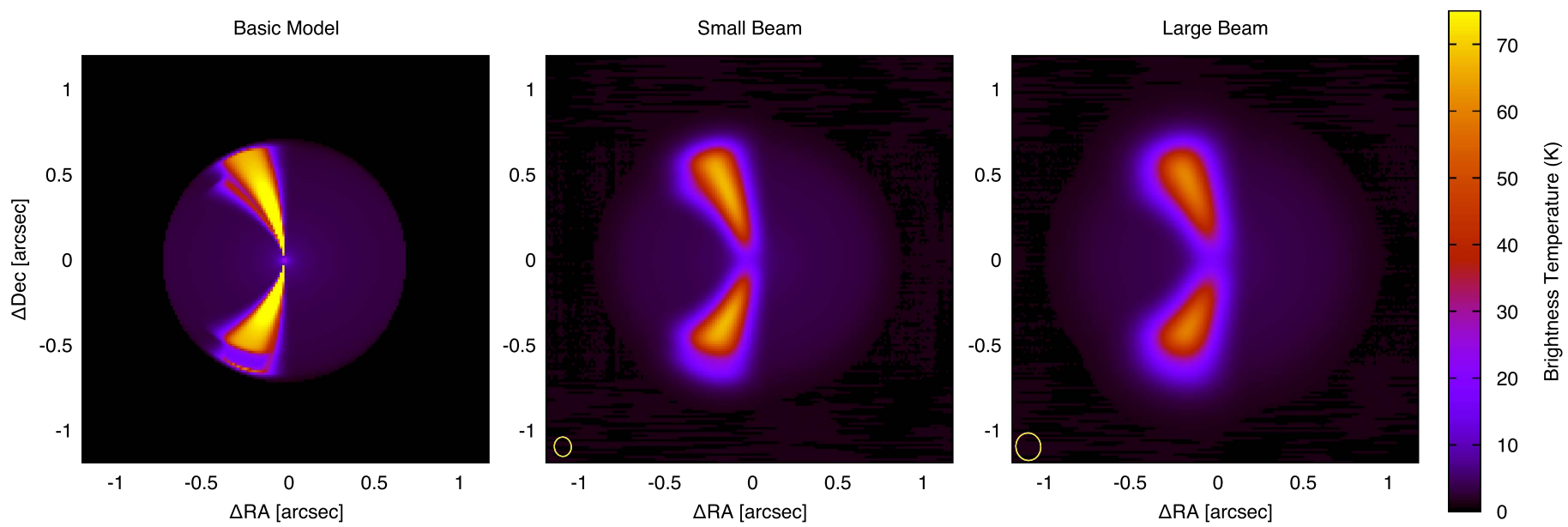

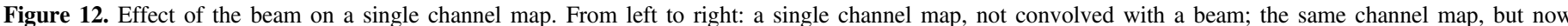

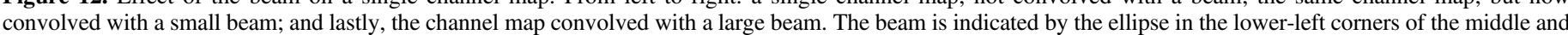
right panels. Color indicates the brightness temperature in Kelvin.

$300 \mathrm{au}$, and a smaller beam size. This means that the channel map size is now much larger than the beam, and both methods work almost equally well.

\subsection{Effect of Finite Sensitivity}

The other issue introduced by actual observation is that of noise. Spatially, noise is typically on the scale of the beam size, and its magnitude depends primarily on the length of the observation. In order to simulate the noise of a real observation such as those described in the previous section, models are processed using the simobserve function of CASA. For the sake of simplicity, we simulated ALMA observations of only the disk model with a mass of $2 \times 10^{-3} M_{\odot}$ (that of Figures 9 , 10 , and 12). As before, we placed the disk a distance of $140 \mathrm{pc}$ from Earth at a decl. of $-24^{\circ}$, and we inclined it by $30^{\circ}$ with respect to the line of sight. Using our ray-tracing code, we generated channel maps of the ${ }^{12} \mathrm{CO} 3-2$ plus dust emission emission between $\pm 21.69 \mathrm{~km} \mathrm{~s}^{-1}$ with a velocity resolution of $0.0145 \mathrm{~km} \mathrm{~s}^{-1}$. The synthetic channel maps were then observed with the simobserve task of CASA adopting antenna configurations 14 and 17, which deliver beam sizes of 0 " $16 \times 0$ !" 14 (for $1 \mathrm{hr}$ synthesis and natural weighting) and 0 " $11 \times 0$ " 09 (for $5 \mathrm{hr}$ synthesis and natural weighting), respectively. The largest recoverable angle scale at the frequency of the line for the chosen configurations is 7 !' 28 and 3 !' 63 , respectively, indicating that the size scale of the disk is smaller than the largest recoverable structure, and no flux is lost. Thermal noise typical of ALMA weather conditions was included, resulting in an rms noise value per channel of $4.2 \mathrm{mJy}^{-1}$ beam $^{-1}$ in the $1 \mathrm{hr}$ long track, and $1.9 \mathrm{mJy}^{\text {beam }}{ }^{-1}$ in the $5 \mathrm{hr}$ long track. CO emission is detected with a peak signal-to-noise ratio of 24 in the higher resolution image and 19 in the lower resolution image.

The top panel of Figure 15 shows a histogram of the ratios of the observed model to the "pure" model for the disk with radius of $100 \mathrm{au}$ and the longer, $5 \mathrm{hr}$ observation. The main result of the noise being added is that the values for the temperature ratio is much more spread out, with most brightness temperatures of the observed model being within $10 \%-15 \%$ of the original, for any given pixel. Given the larger effect of the noise, the differences between using the peak emission map and integrated emission map matter less, though the integrated emission map is still slightly better. The bottom panel of Figure 15 shows the histogram of the temperature ratio, but now for the larger disk. In this case, the two methods give results that are close to the same. It is worth noting that the noise and beam dilution have different effects at different regions of the disk. The beam dilution is mostly a problem at the inner and outer regions, where the noise is applied evenly across the disk. A result of this is that while the beam dilution is less of a problem as the beam gets smaller, the noise is still roughly the same.

This paper has consistently used only the peak emission map, which chooses only the brightest single channel per pixel, and the integrated emission map, which integrates all channels with any detectable line emission. However, these two techniques represent the extremes, and some observations may work best with a compromise between the two. From the standpoint of noise and beam dilution, it is clearly best to average over as many channels as possible, but some of the disks observed in earlier sections clearly benefited from the peak emission map. The best results could feasibly come from a technique halfway between the two, using multiple channels to reduce noise, but restricting to only the brightest few.

\section{Discussion and Conclusions}

This paper has sought to fully explore the techniques and limitations related to calculating a usable brightness temperature from optically thick molecular emission lines in protoplanetary disks. The problem of optically thin emission tends to be significant for observations of fainter lines. This can come about either because the disk in question is not very massive or because the chosen line is not strong. While ${ }^{12} \mathrm{CO}$ has many strong rotational transitions, the rarer isotopologues are considerably fainter. The problem of continuum subtraction, on the other hand, is more of an issue for strong lines and in cases where the continuum emission is also very bright. This can be in particularly massive disks but also in smaller disks that have regions of high dust to gas ratio, such as rings, spirals, or other asymmetries. (See, for example, Pérez et al. 2016; Boehler et al. 2017; Y. Boehler et al. 2017, in preparation.) By introducing peak emission maps as an alternative procedure, the problem of optically thin emission can be avoided and 

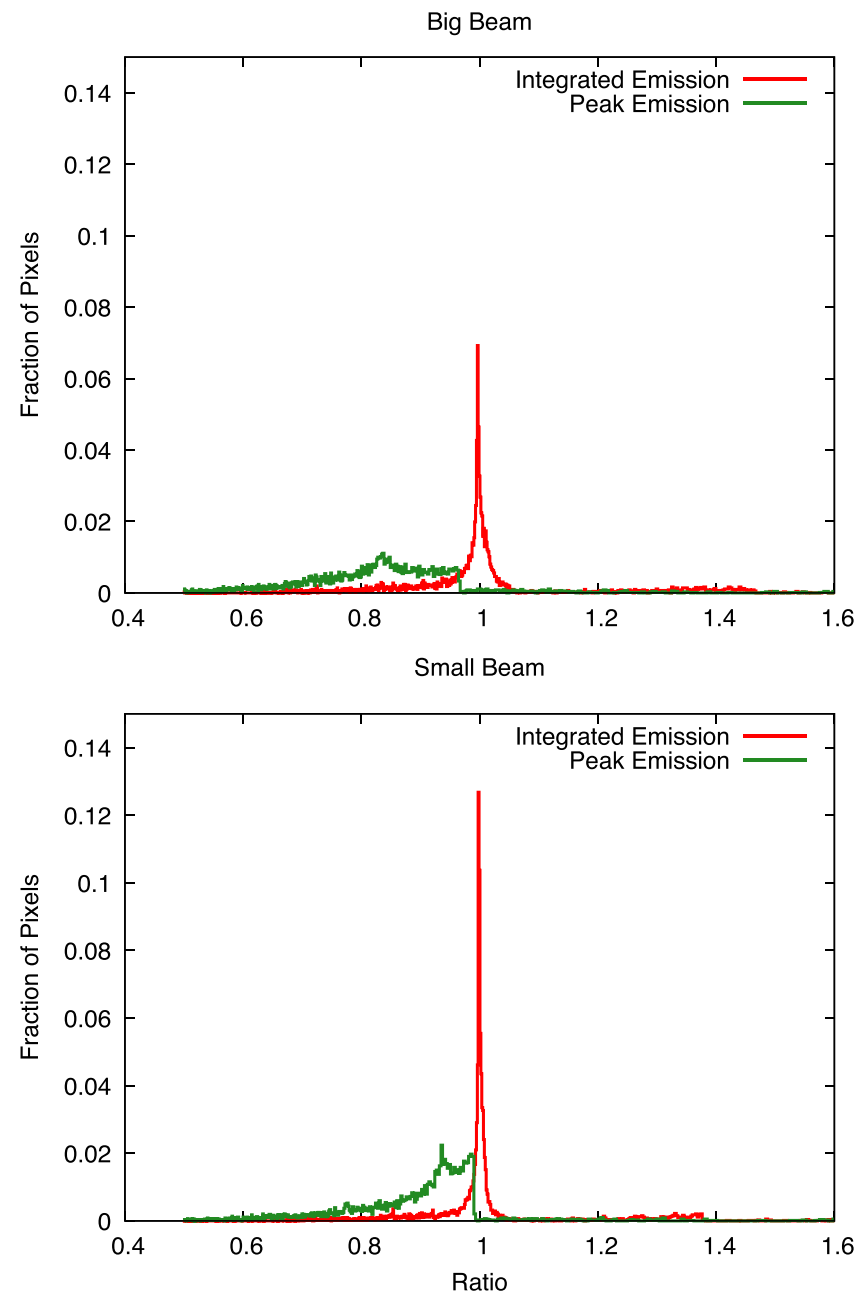

Figure 13. Top panel: ratio of beam-convolved model to non-convolved model for the large beam size. A ratio of 1 indicates that the convolution has no effect, while a higher ratio means that the convolved model has lost flux. While the integrated emission map is largely unaffected, the peak emission map is hardly usable. Beam dilution has removed much of the flux from the image, yielding a brightness temperature artificially low by, on average, $20 \%$. Bottom panel: ratio of beam-convolved model to non-convolved model for the smaller beam size. As before, the integrated emission map is mostly unaffected. Now, however, with a smaller beam, the effects on the peak emission map are better controlled, though still not as good as the integrated emission map. The average loss of flux is now only on the order of $5 \%-10 \%$.

accuracy can be greatly improved by proper handling of continuum subtraction.

The conditions that determine whether to use a peak emission map instead of an integrated emission map and whether or not to subtract the continuum depend on the conditions and choice of target. While there are cases where no choice can yield an accurate brightness temperature, the basic rules are as follows:

1. Lighter disks are more susceptible to the inclusion of optically thin emission from the line wings. The lighter the disk, the better the peak emission map will be relative to the integrated emission map, so long as the peak is optically thick.

2. If both line and continuum are very bright, such as for very massive disks, or for disks with concentrations of

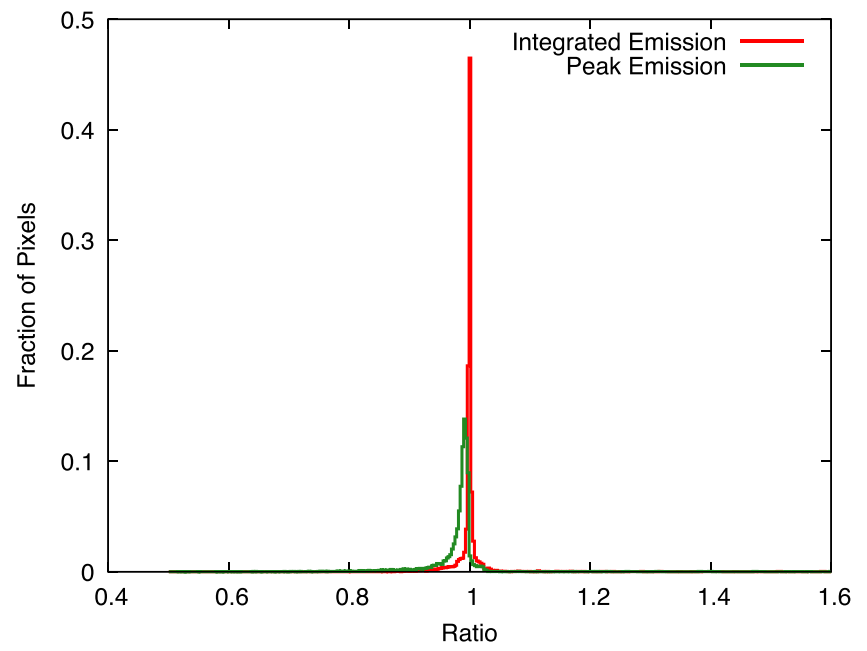

Figure 14. Ratio of beam-convolved model to non-convolved model for the smaller beam size, for a larger disk with radius of 300 au. In this case, the beam size is small enough compared to the channel map scale that both methods adequately recover the non-convolved temperature.
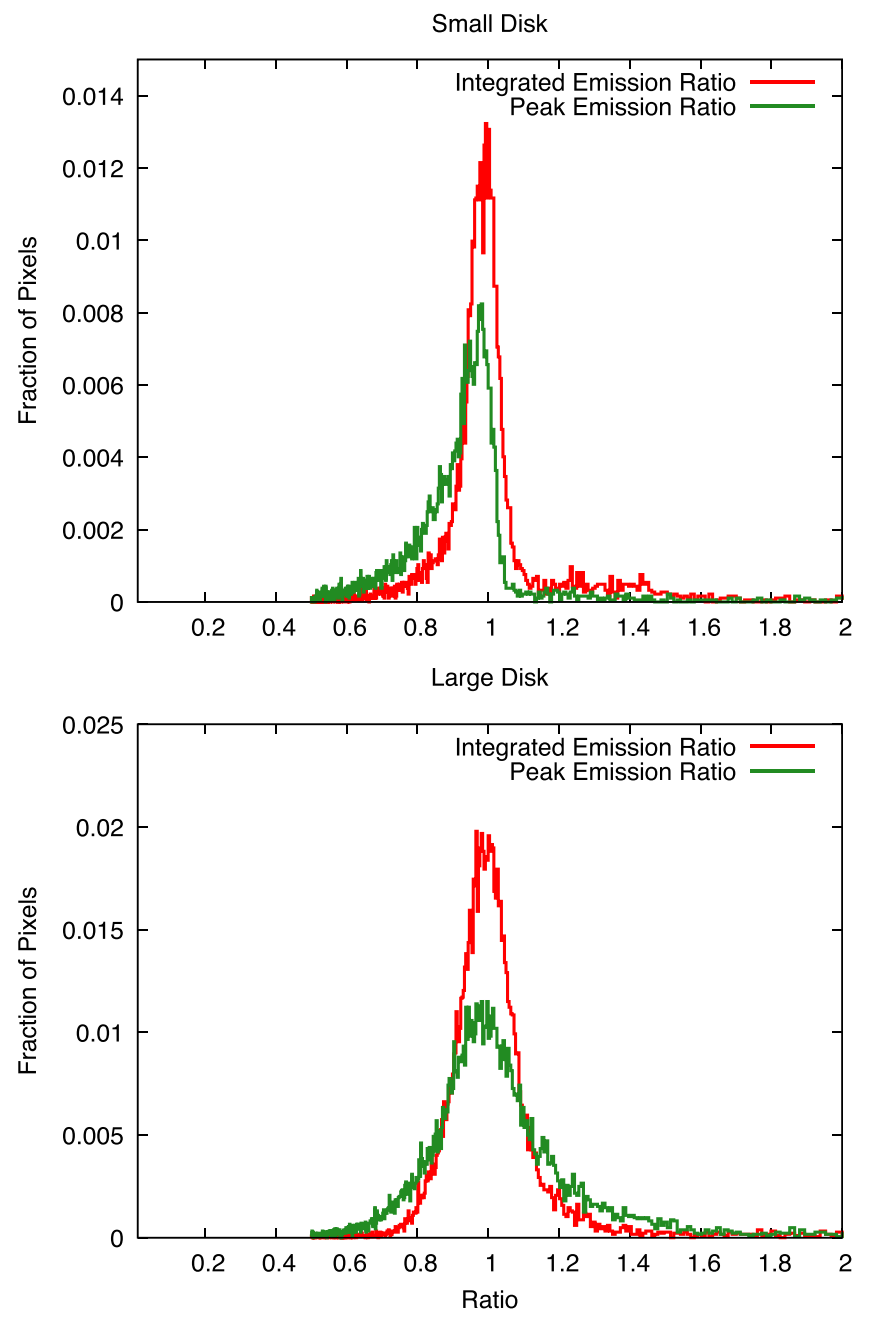

Figure 15. Top panel: ratio of the observed model to the "pure" model for the smaller beam size and smaller disk. With noise added, the observed model is less accurate for both integrated emission map and peak emission map. Bottom panel: ratio of the observed model to the "pure" model for the smaller beam size and larger disk. 
dust, continuum subtraction can remove substantial amounts of flux. It is important to consider if the line could be significantly attenuating the continuum.

3. If the beam size is large relative to the size of the channel maps, the peak emission map will lose substantial flux to beam dilution, while the integrated emission map will not. When the beam size is small, either technique works well.

These conditions are not hard and fast rules, only guidelines. While it is possible to identify regimes where one technique may work better than another, there are usually no strict limits between them. As such, it is necessary that the observer strongly consider the following while analyzing data: first, the optical depth of both the line and the dust, when determining whether or not to subtract the continuum, and if there are any regions of high dust concentration. Second, the strength of the line, when deciding whether to use a peak emission map or integrated emission map. And lastly, the size of the beam and magnitude of the noise. One caveat to this analysis is that measurements of the gas temperature are affected by systematic uncertainties such as absolute flux calibration, which typically ranges between $10 \%$ and $20 \%$.

The observational pitfalls discussed in this paper have several potentially deep effects on future research into protoplanetary disks and planet formation. First, high-resolution disk observations have revealed small-scale structures in the dust distribution (rings and crescents) characterized by surprisingly high dust concentrations. Improper continuum subtraction is a minor effect when continuum emission is negligible, but, as shown in Figure 4, these recently discovered disk structures strongly concentrate dust and therefore emit strong continuum emission that might be optically thick all the way to centimeter wavelengths. See, for example, HD 142725 (Boehler et al. 2017), MWC 758 (Y. Boehler et al. 2017, in preparation), IRS 48 (van der Marel et al. 2015), HL Tau (Andrews et al. 2016), and HD 163296 (Isella et al. 2016). Key information about the nature of these structures, which is still debated, can be acquired by measuring the amount and temperature of molecular gas using optically thin and optically thick lines, respectively. In both cases, the interpretation of the observations requires considerable caution because line and continuum emission mutually interact, absorbing each other. As shown in the previous sections, the standard procedure for continuum subtraction can lead to a lack of gas emission from regions where the continuum emission is strong. This, in turn, can lead to erroneous estimates of both the gas column density and temperature, and consequently, to erroneous physical interpretations. For example, the lack of $\mathrm{CO}$ emission could be interpreted as the result of the freeze-out of molecules on dust grains. The underestimate of the $\mathrm{CO}$ luminosity/temperature might also have an effect on the calculation of the disk turbulence because fainter, and therefore colder, lines could result in the gas turbulence velocity being overestimated. However, since the turbulence is derived in practice by comparing the line intensity with the line broadening (see Flaherty et al. 2015, 2017), it is unclear what the combined effect of mutual absorption between gas and dust might be. The derivation of turbulence from $\mathrm{CO}$ observations will be discussed in a future paper.

Additionally, ALMA surveys of nearby star-forming regions are delivering a large amount of observation of dust and $\mathrm{CO}$ emission from nearby protoplanetary disks (see, for example, Ansdell et al. 2017). The observations could be statistically interpreted in order to estimate the disk masses. See Barenfeld et al. (2016) or Manara et al. (2016). Some of the effects shown here (such as continuum subtraction) may have an impact on the derivation of gas mass from line intensities. In particular, since much gas mass can be hidden in the central and more optically thick regions, continuum subtraction, and, to the same extent, beam dilution, could lead to the disk mass being systematically underestimated. This effect will increase with the disk mass. Statistical interpretation of the line emission and line emission ratios should therefore properly take into account both the continuum opacity and the effect of continuum subtraction on the observations.

Lastly, it is worth mentioning that this paper considered the techniques of determining temperature from single emission lines. In many areas of astronomy, an alternate method is often employed to determine temperatures. For a given atom or molecule in LTE, the populations of different levels are given by Boltzmann statistics, so the ratio of two lines will be a function only of the temperature. This technique was employed, for example, by Dartois et al. (2003) and Fedele et al. (2016). However, this technique is not optimal for the applications discussed in this paper for several reasons. First, and most importantly, it is more difficult from an observational standpoint because it requires multiple lines to be observed. Attempting to apply this technique to rotational modes of $\mathrm{CO}$, for example, would require multiple separate observations, since the multiple transitions rarely fall within any given band. Multiple observations are more expensive and then must be separately calibrated. Since the frequency of the two observations varies significantly, the beam size will be different as well. Second, the error coming from multiple observations will be higher than that for a single observation. In general, the errors will add in quadrature, and the already potentially significant systematic errors can become much more problematic. Lastly, from a physical standpoint, the temperature resulting from this method is hard to interpret in the case of optically thick emission. Unlike for optically thin observation, where the brightness temperature describes a bulk property of the gas along the entire line of sight, each observation of an optically thick line will correspond to an emission region at a different depth in the disk since the opacity depends strongly on the frequency of observation. As circumstellar disks have substantial temperature variation, both vertically and radially, these regions will likely have different temperatures and compositions, so a value determined from both may not meaningfully correspond to either. Using line ratios to derive a temperature does, however, offer the advantage that the lines observed do not need to be optically thick in order to determine a temperature. This makes it potentially useful for extremely light disks, where even ${ }^{12} \mathrm{CO}$ is optically thin.

Y.B. and A.I. acknowledge support from the NASA Origins of Solar Systems program through award NNX15AB06G. A.I. acknowledges support from NSF grant Nos. AST-1535809 and AST-1715718. E.W. acknowledges support from the NRAO Student Observing Support grant Nos. AST-0836064 NSF and AST-1519126. 


\section{Appendix A Integration of the Radiative Transfer Equation}

In most cases, the synthetic emission from a circumstellar disk must be calculated via numerical integration of the radiative transfer equation, given a density profile, temperature profile, and requisite opacities. This process can be complicated and computationally expensive. So, it is useful, at least for pedagogical purposes, to consider the case of a face-on, vertically isothermal, axisymmetric disk, for the simple reason that it presents a case where the radiative transfer equation becomes analytically solvable. In the following derivations, $r$ is the cylindrical radius and $z$ is the vertical distance from the disk midplane.

In the relative low density of protoplanetary disks, and for the range of frequencies considered, scattering is negligible (but see Kataoka et al. 2015), and the radiative transfer equation reduces to

$$
\frac{d I_{\nu}}{d s}=K_{\nu}(s)\left[B_{\nu}(T(s))-I_{\nu}(s)\right]
$$

where $K_{\nu}(s)=\kappa_{\nu}(s), \rho(s)$ is the opacity at a given point, and $s$ is the spatial coordinate along the line of sight. This assumes that the system is in local thermodynamic equilibrium. As discussed in Section 2.1, however, LTE is a reasonable assumption for the case of $\mathrm{CO}$ rotational lines at these temperatures.

In the special case of a face-on disk, rays propagate through the disk aligned with the $z$-axis, which means integration only needs to be done along this axis, allowing the differential equation to be solved analytically. Because we have chosen a vertically isothermal disk structure, $K_{\nu}$ explicitly depends only on $r$. Inserting the density profile into Equation (5) gives

$$
\frac{d I}{d z}=\frac{\kappa_{\nu}(r) \cdot \Sigma(r)}{\sqrt{2 \pi} h(r)} \exp \left(\frac{-z^{2}}{2 h^{2}(r)}\right)\left(B_{\nu}(T)-I(z)\right) .
$$

This equation is separable,

$$
\frac{d I}{B_{\nu}(T)-I(z)}=\frac{\kappa_{\nu}(r) \cdot \Sigma(r)}{\sqrt{2 \pi} h(r)} \exp \left(\frac{-z^{2}}{2 h^{2}(r)}\right) d z .
$$

Integrating both sides yields

$$
\begin{aligned}
-\ln \left(B_{\nu}(T)-I(z)\right)= & \frac{1}{2} \frac{\kappa_{\nu}(r) \cdot \Sigma(r)}{\sqrt{2 \pi} h(r)} \sqrt{2 \pi} h(r) \\
& \cdot \operatorname{erf}\left(\frac{z}{\sqrt{2 h^{2}(r)}}\right)+C,
\end{aligned}
$$

which, after cancellation and exponentiation, becomes

$$
I(z)=C \cdot \exp \left(-\frac{\kappa_{\nu}(r) \cdot \Sigma(r)}{2} \operatorname{erf}\left(\frac{z}{\sqrt{2} h(r)}\right)\right)+B_{\nu}(T)
$$

Using the initial condition that $I=0$ at the starting location, $z_{\text {in }}$, we can solve for the constant of integration:

$$
C=-B_{\nu}(T) \exp \left(\frac{\kappa_{\nu}(r) \cdot \Sigma(r)}{2} \operatorname{erf}\left(\frac{z_{\text {in }}}{\sqrt{2} h(r)}\right)\right)
$$

The final result is quite complicated but not intractable:

$$
\begin{aligned}
I= & B_{\nu}(T(r))\left(1-\exp \left(\frac { 1 } { 2 } \kappa _ { \nu } ( r ) \cdot \Sigma ( r ) \left(\operatorname{erf}\left(\frac{z_{\text {in }}}{\sqrt{2} h(r)}\right)\right.\right.\right. \\
& \left.\left.-\operatorname{erf}\left(\frac{z_{\text {out }}}{\sqrt{2} h(r)}\right)\right)\right),
\end{aligned}
$$

where $z_{\text {in }}$ and $z_{\text {out }}$ are the limits of integration along the line of sight. In most cases, it is practical to define outer boundaries for the disk, but to good approximation, we can treat the disk as infinite in vertical extent and replace the start and end points with positive and negative infinity. This simplifies the final solution to the more manageable

$$
I=B_{\nu}(T)\left(1-\exp \left(-\kappa_{\nu}(r) \cdot \Sigma(r)\right)\right)
$$

The optical depth for a given line of sight can be calculated by a similar process,

$$
\begin{gathered}
\tau=\int K_{\nu}(s) \rho(s) d s=\kappa_{\nu}(r) \int \frac{\Sigma(r)}{\sqrt{2 \pi} h(r)} \exp \left(\frac{-z^{2}}{2 h^{2}(r)}\right) d z \\
\tau=\kappa_{\nu}(r) \frac{\Sigma(r)}{\sqrt{2 \pi} h(r)} \cdot \frac{1}{2} \sqrt{2 \pi} \cdot \operatorname{erf}\left(\frac{z}{\sqrt{2} h(r)}\right)+C
\end{gathered}
$$

Using the initial condition that the optical depth is zero at the starting point, the constant of integration can be calculated, and the final result is

$$
\tau=\frac{\kappa_{\nu}(r) \cdot \Sigma(r)}{2}\left(\operatorname{erf}\left(\frac{z_{\text {out }}}{\sqrt{2} h(r)}\right)-\operatorname{erf}\left(\frac{z_{\text {in }}}{\sqrt{2} h(r)}\right)\right)
$$

This can be simplified using the approximation that $z_{\text {in }}$ and $z_{\text {out }}$ are sufficiently large, yielding

$$
\tau=\kappa_{\nu}(r) \cdot \Sigma(r) .
$$

The derivation of Equation (11) follows by a process roughly analogous to the previous cases, except that now, the opacity of the emitting material and the opacity of the absorbing material differ. To calculate the attenuated continuum, the emitting opacity is only that of the dust, while the absorbing opacity is that of both the dust and the gas. As such, the radiative transfer equation reduces to

$$
\frac{d I_{\nu}}{d s}=\rho(s)\left[\kappa_{\nu, \mathrm{emit}} \cdot B_{\nu}(T(s))-\kappa_{\nu, \mathrm{abs}} \cdot I_{\nu}(s)\right] .
$$

As before, the line of sight is going straight down, and the path is entirely isothermal. Thus, the intensity and temperature depend only on the radius. Plugging in the density profile gives

$$
\frac{d I_{\nu}(z)}{d s}=\frac{\Sigma(r) \kappa_{\nu, \mathrm{abs}}}{\sqrt{2 \pi} h(r)} \cdot \exp \left(\frac{-z^{2}}{2 h^{2}(r)}\right)\left(\frac{\kappa_{\nu, \mathrm{emit}}}{\kappa_{\nu, \mathrm{abs}}} B_{\nu}(T(r))-I_{\nu}(z)\right) .
$$

The equation can be separated,

$$
\frac{d I_{\nu}(z)}{\frac{\kappa_{\nu, \mathrm{em}}}{\kappa_{\nu, \mathrm{abs}}} B_{\nu}(T(r))-I_{\nu}(z)}=\frac{\Sigma(r) \kappa_{\nu, \mathrm{abs}}}{\sqrt{2 \pi} h(r)} \cdot \exp \left(\frac{-z^{2}}{2 h^{2}(r)}\right) d z .
$$


Integrating both sides yields

$$
\begin{aligned}
& -\ln \left(\frac{\kappa_{\nu, \mathrm{em}}}{\kappa_{\nu, \mathrm{abs}}} B_{\nu}(T(r))-I_{\nu}(z)\right) \\
& =\frac{\sum(r)}{2} \kappa_{\nu, \mathrm{abs}} \cdot \operatorname{erf}\left(\frac{z}{\sqrt{2} h(r)}\right)+C,
\end{aligned}
$$

where $C$ is a constant of integration. Thus,

$$
\begin{aligned}
I_{\nu}(z)= & \frac{\kappa_{\nu, \mathrm{em}}}{\kappa_{\nu, \mathrm{abs}}} B_{\nu}(T(r))-C \cdot \exp \left(-\frac{\Sigma(r)}{2} \kappa_{\nu, \mathrm{abs}}\right. \\
& \left.\cdot \operatorname{erf}\left(\frac{z}{\sqrt{2} h(r)}\right)\right) .
\end{aligned}
$$

We can use the initial condition that $I_{\nu}\left(z_{\text {in }}\right)=0$ to solve for the constant of integration:

$$
C=\frac{\kappa_{\nu, \mathrm{em}}}{\kappa_{\nu, \mathrm{abs}}} B_{\nu}(T(r)) \cdot \exp \left(\frac{\Sigma(r)}{2} \kappa_{\nu, \mathrm{abs}} \cdot \operatorname{erf}\left(\frac{z_{\text {in }}}{\sqrt{2} h(r)}\right)\right) .
$$

Plugging this value in and simplifying gives

$$
\begin{aligned}
I_{\nu}(z)= & \frac{\kappa_{\nu, \mathrm{em}}}{\kappa_{\nu, \mathrm{abs}}} B_{\nu}(T(r))\left(1-\exp \left(\frac { \Sigma ( r ) } { 2 } \kappa _ { \nu , \mathrm { abs } } \left(\operatorname{erf}\left(\frac{z_{\text {in }}}{\sqrt{2} h(r)}\right)\right.\right.\right. \\
& \left.\left.-\operatorname{erf}\left(\frac{z}{\sqrt{2} h(r)}\right)\right)\right) .
\end{aligned}
$$

Using the simplifying assumption that the ray begins at minus infinity and goes to infinity, we recover Equation (11),

$$
I_{\nu}(z)=\frac{\kappa_{\nu, \mathrm{em}}}{\kappa_{\nu, \mathrm{abs}}} B_{\nu}(T(r))\left(1-\exp \left(-\Sigma(r) \cdot \kappa_{\nu, \mathrm{abs}}\right)\right) .
$$

\section{Appendix B CO Opacity Model}

The opacity of a given point in space is the sum of the continuum opacity and $\mathrm{CO}$ opacity. Continuum opacity must be calculated using Mie Theory, for which several programs already exist. CO opacity is simpler and can be derived relatively easily from first principles. Broadly speaking, for a transition between the states $i$ and $j$, the energy $h \nu_{0}$ is absorbed from the radiation $I_{\nu}$, where $\nu_{0}$ is the rest energy of the transition. The relation is given by

$$
\kappa_{i j} I_{\nu} d \nu d \Omega=h \nu_{0}\left(n_{i} \phi_{\nu} B_{i j} I_{\nu}-n_{j} \psi_{\nu} B_{j i} I_{\nu}\right) \frac{d \nu d \Omega}{4 \pi},
$$

where $\kappa_{i j}$ is the mass absorption coefficient, $\phi_{\nu}$ and $\psi_{\nu}$ are the absorption and emission profiles, and $B_{j i}$ and $B_{i j}$ are the Einstein coefficients for stimulated emission and photoabsorption. The shape of the profiles can vary but must always be normalized to unity. Solving for the mass absorption coefficient gives

$$
\kappa_{i j}=\frac{h \nu_{0} n_{i} \phi_{\nu} B_{i j}}{4 \pi}\left(1-\frac{n_{j} \psi_{\nu} B_{j i}}{n_{i} \phi_{\nu} B_{i j}}\right) .
$$

This can be simplified using one of the Einstein relations,

$$
g_{i} B_{i j}=g_{j} B_{j i},
$$

and, under the conditions of LTE, Boltzmann statistics relates the populations through

$$
\frac{n_{j}}{n_{i}}=\frac{g_{j}}{g_{i}} \exp \left(-\frac{h \nu_{0}}{k T}\right)
$$

to give

$$
\kappa_{i j}=\frac{h \nu_{0} n_{i} \phi_{\nu} B_{i j}}{4 \pi}\left(1-\exp \left(-\frac{h \nu_{0}}{k T}\right)\right)
$$

Here, we have also used the assumption of complete redistribution, which means that the absorption and emission profiles are the same. This expression is most frequently expressed using the Einstein $A$ value, related through the Einstein relation

$$
A_{j i}=\frac{2 h \nu}{c^{2}} B j i
$$

to obtain

$$
\kappa_{i j}=\frac{c^{2}}{8 \pi \nu_{0}^{2}} \cdot \frac{g_{j}}{g_{i}} n_{i} \phi_{\nu} A_{j i}\left(1-\exp \left(-\frac{h \nu_{0}}{k T}\right)\right) .
$$

The number of molecules of $\mathrm{CO}$ currently in state $i, n_{i}$, is given by the Boltzmann equation,

$$
n_{i}=n_{C O} \cdot \frac{g_{i} \exp \left(-\frac{E_{i}}{k T}\right)}{Z(T)},
$$

where $n_{\mathrm{CO}}$ is the number of $\mathrm{CO}$ particles per gram of gas, $E_{i}$ is the energy of the level, and $Z(T)$ is the partition function. For rotational transitions, the partition function can be expressed as

$$
Z(T)=\sum_{i=0}^{\infty}(2 i+1) \exp \left(\frac{-E_{i}}{k T}\right)
$$

The partition function can also be evaluated quickly and with high accuracy via a power series expansion:

$$
\begin{aligned}
Z(T)= & \frac{k T}{h B_{0}}+\frac{1}{3}+\frac{1}{15}\left(\frac{h B_{0}}{k T}\right)+\frac{4}{315}\left(\frac{h B_{0}}{k T}\right)^{2} \\
& +\frac{1}{315}\left(\frac{h B_{0}}{k T}\right)^{3}+\ldots,
\end{aligned}
$$

where $B_{0}$ is the rigid rotor rotation constant for the molecule. For more details, see Mangum \& Shirley (2015). $\phi_{\nu}$ is the natural line profile and is given by

$$
\phi_{\nu}=\frac{c}{\nu_{0}} \cdot \frac{1}{\Delta V \sqrt{\pi}} \exp \left(-\frac{\Delta^{2} \nu}{\Delta^{2} V}\right),
$$

with $\Delta \nu$ being the line-of-sight velocity of the gas. This is a Gaussian profile, typical of thermalized gas, but is generalized to include both thermal broadening and possible turbulent broadening, both included in

$$
\Delta V=\sqrt{\frac{2 k T}{m_{\mathrm{CO}}}+V_{\mathrm{turb}}^{2}} .
$$


While the equations above are fully general, the calculations done in this paper assume there is no turbulence in the gas. Though other sources of broadening exist, none are particularly relevant under these conditions. Furthermore, in several of the cases considered in this paper, the disk is viewed face on, and the line-of-sight velocity resulting from Keplerian rotation is zero.

The Einstein $A$ value can, in principle, be calculated from theoretical models. Mihalas gives the Einstein $A$ value for dipole emission as

$$
A_{j i}=\frac{64 \pi^{4} \nu^{3}}{3 h c^{3}}|\mu|^{2}
$$

where $|\mu|^{2}$ is the squared dipole matrix element summed over degenerate states, and can in this case be expressed as

$$
\left|\mu_{j i}\right|^{2}=\frac{j+1}{2 j+1} \mu^{2},
$$

with $\mu$ being the permanent dipole moment of the molecule. (See Hubeny \& Mihalas 2015, or Spitzer 1978 for further details.) It is worth noting, however, that the theoretical values do not agree particularly well with experimentally measured values, such as those from the Leiden LAMDA database. This paper, therefore, uses the measured values instead.

\section{Appendix C \\ Ray Tracing}

While Appendix A described an analytical solution to the radiative transfer equation, it was predicated on several simplifying assumptions. The disk structure was taken to be vertically Gaussian, the temperature vertically isothermal, and most importantly, the line of sight was also vertical. While useful as a proof of concept, this model is far too simple to be sufficient. However, adding any further details complicates the problem enough that no analytical solution is viable. Toward that end, this paper uses a new, numerical ray-tracing code to analyze more realistic disk models.

The code is written entirely in $\mathrm{C}++$, using the new language features and libraries introduced with the $\mathrm{C}++11$ standard. Unlike other commonly used ray-tracing codes (for example, RADMC-3D), it does not use a gridded disk structure. Gridded models work by dividing the disk into radius, zenith, and azimuth bins, each with a separate density, temperature, and composition. Ray tracing is then done by casting a ray through the disk and summing the contribution and attenuation due to each grid cell the ray passes through. This approach has several advantages. First, the output of most radiative transfer codes is in the same gridded form, because radiative transfer of this form must be done via Monte Carlo methods. Second, the calculation of the emission is very simple. The primary disadvantage is that the gridded approach is slowed down by the overhead of calculating the correct grid cells to include. Determining which cell to enter requires calculating the intersections with all nearby edges, which is nontrivial, and leads to numerical instabilities at the corners. Additionally, while easy to implement, this is a first-order method of solving the radiative transfer equation. This makes it well-behaved but extremely slow.

The code used in this paper opts to instead use a nongridded approach. This means that input files, such as the temperature, must be interpolated on, but this does not add much computational time. Considerable time is saved by removing all of the geometric calculations. Furthermore, the differential equation does not need to be solved with a firstorder method. In theory, this means a higher order adaptive algorithm (such as the commonly used combined fourthfifth-order Runge-Kutta scheme and its higher order replacements), but in practice the radiative transfer equation is difficult to numerically solve. Almost all emission is generated from a region around the $\tau=1$ surface: in front of this region, the density is low enough that there is minimal emission, and behind it, the opacity is high enough that any emission is attenuated away. Simple numerical integration schemes that approximate the solutions to differential equations as smooth and low-order polynomials have difficulty with such a sharp solution. Additionally, adaptive step size algorithms increase their steps in larger and larger amounts as they move through the initial region, and then usually completely overstep the emission region, giving a completely wrong final result. In the future, it may be possible to work around these problems, but for the present time, we are restricted to the implicit trapezoid rule instead. There are several reasons for this: most importantly, the radiative transfer equation without scattering, as written in Equation (5), is a stiff equation. An equation is stiff if its exact solution has a term that decays as an exponential, but has derivatives that are larger than the terms they correspond to. Stiff equations cannot be solved with explicit numerical schemes, because these algorithms will diverge. Instead, implicit techniques must be used. Second, the trapezoid rule is the highest order integration scheme that is absolutely convergent. This means that it will converge to the correct solution regardless of the step size. Though it does not converge as quickly as a higher order method would, it is guaranteed to converge to the correct solution, unlike other methods.

In practice, the use of a non-gridded disk with the implicit trapezoid rule offers a speed boost of roughly twice that of the other methods tested (mainly RADMC-3D). This boost in speed is made possible possible primarily by the fact that this code is highly optimized for this specific problem, where other codes like RADMC-3D are designed to be much more general and can handle other geometries, or even include scattering. In order to further increase the efficiency of the simulations, the code is multithreaded, which allows the speed to scale linearly with the number of CPU cores allotted.

The image is constructed as an array of pixels, each corresponding to a line of sight cast out normal to the image surface. All imaging is done under the assumption that the disk is so distant that the small angle approximation is valid and the lines of sight are approximately parallel, a reasonable assumption, given that even the closest disks are still dozens of parsecs away. Imaging is multithreaded so that multiple rays can be done in parallel.

Results from disk imaging have been tested for accuracy against both the analytical solution described in Appendix A as well as the other codes written for similar processes, such as RADMC-3D and RADEX. Results match the analytical solution almost exactly and are close to matching output from other ray-tracing codes. In terms of speed, this approach is considerably faster than other available ray-tracing codes, both for single-processor comparisons and through being 
Radial Intensity

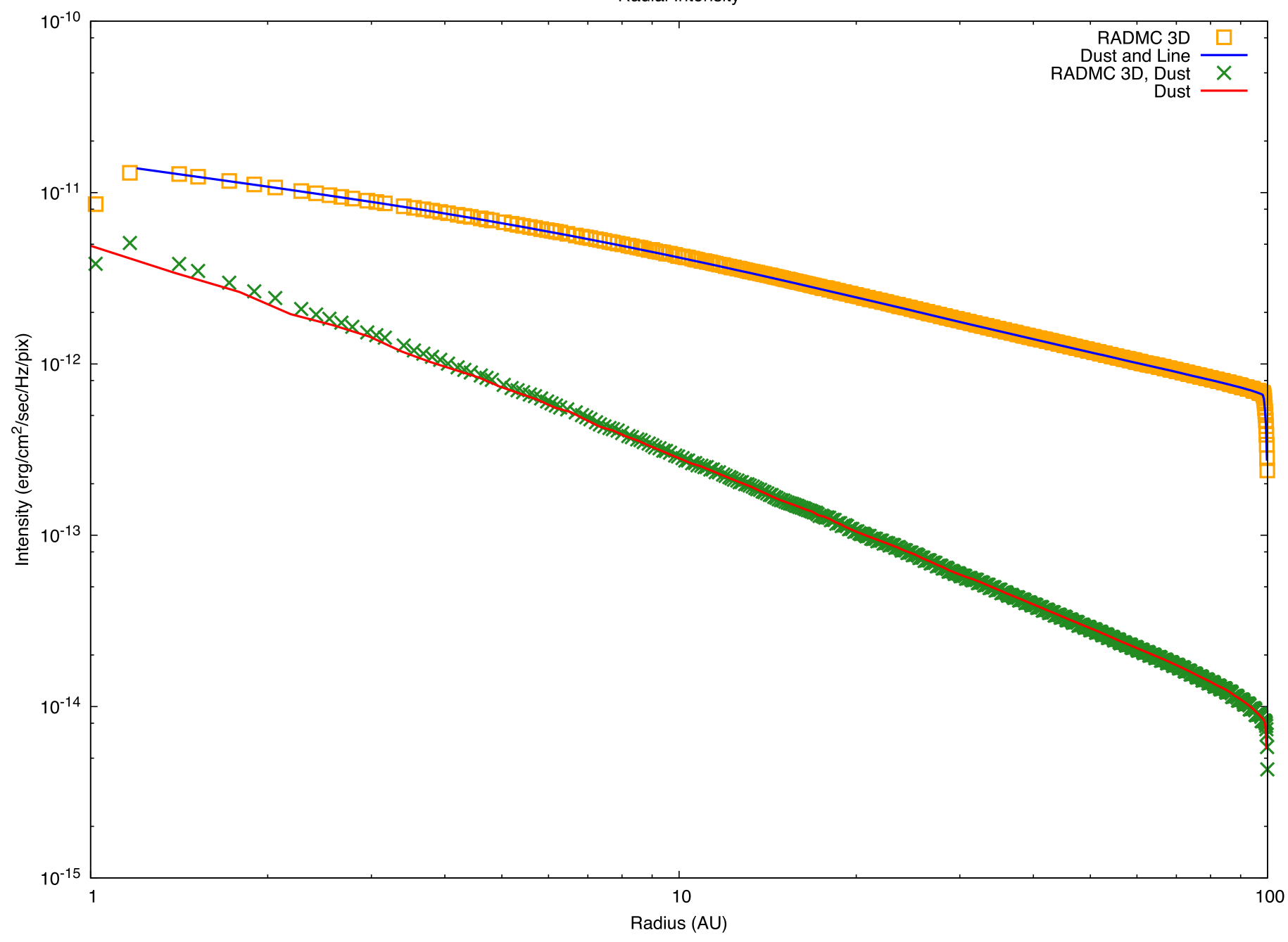

Figure 16. Agreement between outputs from the code used in this paper and RADMC-3D. The simulation in this case is a face-on disk with a mass of $2 \times 10^{-2} M_{\odot}$, with an outer radius of 100 au. Two sets of data are presented: blue and orange correspond to the models including both continuum and line emission, whereas red and green correspond to the models that contain only continuum emission. The lines are the output of the code used in this paper, whereas the points are the results from RADMC-3D.

multithreaded. Figure 16 shows the matching in results between the code used for this paper and the output for an identical disk model from RADMC-3D. The model shown is a disk of mass $2 \times 10^{-2} M_{\odot}$, with an outer radius of $100 \mathrm{au}$. Two sets of data are presented: blue and orange correspond to the models including both continuum and line emission, whereas red and green correspond to the models that contain only continuum emission. The lines are the output of the code used in this paper, whereas the points are the results from RADMC-3D. The agreement is quite close, despite the fundamentally different integration schemes.

\section{ORCID iDs}

Andrea Isella (ib https://orcid.org/0000-0001-8061-2207 Yann Boehler (i) https://orcid.org/0000-0002-8692-8744

\section{References}

Andrews, S. M., Wilner, D. J., Zhu, Z., et al. 2016, ApJL, 820, L40 Ansdell, M., Williams, J. P., Manara, C. F., et al. 2017, AJ, 153, 240 Barenfeld, S. A., Carpenter, J. M., Ricci, L., \& Isella, A. 2016, ApJ, 827, 142 Beckwith, S. V. W., \& Sargent, A. I. 1993, ApJ, 402, 280
Bergin, E. A., Du, F., Cleeves, L. I., et al. 2016, ApJ, 831, 101

Blum, J., \& Wurm, G. 2008, ARA\&A, 46, 21

Boehler, Y., Weaver, E., Isella, A., et al. 2017, ApJ, 840, 60

Casassus, S., van der Plas, G., M, S. P., et al. 2013, Natur, 493, 191

Chiang, E. I., \& Goldreich, P. 1997, ApJ, 490, 368

Condon, J. J., \& Ransom, S. M. 2016, Essential Radio Astronomy (Princeton, NJ: Princeton Univ. Press)

Dartois, E., Dutrey, A., \& Guilloteau, S. 2003, A\&A, 399, 773

Dubernet, M. L., et al. 2012, A\&A, 553, 50

Dullemond, C. 2012, Astrophysics Source Code Library, RADMC-3D, ascl:1202.015

Dullemond, C. P., Dominik, C., \& Natta, A. 2001, ApJ, 560, 957

Dullemond, C. P., Hollenbach, D., Kamp, I., \& D'Alessio, P. 2007, Protostars and Planets V (Tucson, AZ: Univ. Arizona Press), 555

Dullemond, C. P., van Zadelhoff, G. J., \& Natta, A. 2002, A\&A, 389, 464

Fedele, D., van Dishoeck, E. F., Kama, M., Bruderer, S., \& Hogerheijde, M. R. 2016, A\&A, 591, A95

Flaherty, K. M., Hughes, A. M., Rose, S. C., et al. 2017, ApJ, 843, 150

Flaherty, K. M., Hughes, A. M., Rosenfeld, K. A., et al. 2015, ApJ, 813, 99

Guilloteau, S., Di Folco, E., Dutrey, A., et al. 2013, A\&A, 549, A92

Hubeny, I., \& Mihalas, D. 2015, Theory of Stellar Atmospheres (Princeton, NJ: Princeton Univ. Press), 221

Isella, A., Carpenter, J. M., \& Sargent, A. I. 2009, ApJ, 701, 260

Isella, A., Guidi, G., Testi, L., et al. 2016, PhRvL, 117, 25

Isella, A., Testi, L., Natta, A., et al. 2007, A\&A, 469, 213

Jin, S., Li, S., Isella, A., Li, H., \& Ji, J. 2016, ApJ, 818, 76

Juvela, M., Harju, J., Ysard, N., \& Lunttila, T. 2012, A\&A, 538, A133 
Kama, M., Bruderer, S., Carney, M., et al. 2016, A\&A, 588, A108

Kataoka, A., Muto, T., Momose, M., et al. 2015, ApJ, 809, 78

Manara, C. F., Rosotti, G., Testi, L., et al. 2016, A\&A, 591, L3

Mangum, J. G., \& Shirley, Y. L. 2015, PASP, 127, 266

Öberg, K. I., Boogert, A. C. A., Pontoppidan, K. M., et al. 2011, ApJ, 740, 109

Pérez, L. M., Carpenter, J. M., Andrews, S. M., et al. 2016, Sci, 353, 1519

Piétu, V., Dutrey, A., \& Guilloteau, S. 2007, A\&A, 467, 163

Pollack, J. B., Hollenbach, D., Beckwith, S., et al. 1994, ApJ, 421, 615

Qi, C., Öberg, K. I., Wilner, D. J., et al. 2013, Sci, 341, 630

Rafikov, R. R., \& De Colle, F. 2006, ApJ, 646, 275

Rosenfeld, K. A., Andrews, S. M., Hughes, A. M., Wilner, D. J., \& Qi, C. 2013, ApJ, 774, 16

Schwarz, K. R., Bergin, E. A., Cleeves, L. I., et al. 2016, ApJ, 823, 91
Spitzer, L. 1978, Physical Processes in the Interstellar Medium (Princeton, NJ: Princeton Univ. Press)

Teague, R., Guilloteau, S., Semenov, D., et al. 2016, A\&A, 592, A49

Testi, L., Birnstiel, T., Ricci, L., et al. 2014, in Protostars and Planets VI, ed. H. Beuther et al. (Tucson, AZ: Univ. Arizona Press), 339

van der Marel, N., Pinilla, P., Tobin, J., et al. 2015, ApJL, 810, L7

van der Marel, N., van Dishoeck, E. F., Bruderer, S., et al. 2016, A\&A, 585, A58

van der Plas, G., Casassus, S., Ménard, F., et al. 2014, ApJL, 792, L25

van Zadelhoff, G.-J., van Dishoeck, E. F., Thi, W.-F., \& Blake, G. A. 2001, A\&A, 377, 566

Williams, J. P., \& Best, W. M. J. 2014, ApJ, 788, 59

Williams, J. P., \& Cieza, L. A. 2011, ARA\&A, 49, 67

Yen, H.-W., Liu, H. B., Gu, P.-G., et al. 2016, ApJ, 820, L25 